\title{
Loss of Arabidopsis GAUT12/IRX8 causes anther indehiscence and leads to reduced G lignin associated with altered matrix polysaccharide deposition
}

\author{
Zhangying Hao ${ }^{1,2,3}$, Utku Avci ${ }^{2,3}$, Li Tan ${ }^{2,3}$, Xiang Zhu ${ }^{2,4}$, John Glushka ${ }^{2}$, Sivakumar Pattathil ${ }^{2,3}$, \\ Stefan Eberhard ${ }^{2}$, Tipton Sholes ${ }^{2}$, Grace E. Rothstein ${ }^{2,5}$, Wolfgang Lukowitz ${ }^{1}$, Ron Orlando ${ }^{2,4}$, \\ Michael G. Hahn ${ }^{1,2,3}$ and Debra Mohnen ${ }^{2,3,6 *}$
}

${ }^{1}$ Department of Plant Biology, University of Georgia, Athens, GA, USA

${ }^{2}$ Complex Carbohydrate Research Center, University of Georgia, Athens, GA, USA

${ }^{3}$ BioEnergy Science Center (BESC), Oak Ridge National Laboratory, Oak Ridge, TN, USA

${ }^{4}$ Department of Chemistry, University of Georgia, Athens, GA, USA

${ }^{5}$ Department of Biology, Lawrence University, Appleton, WI, USA

${ }^{6}$ Department of Biochemistry and Molecular Biology, University of Georgia, Athens, GA, USA

Edited by:

Cécile Albenne, Université Paul Sabatier Toulouse (UPS), France

\section{Reviewed by:}

Henrik Scheller, Lawrence Berkeley National Laboratory, USA

Kian Hématy, Institut National de la

Recherche Agronomique, France

Rui Shi, North Carolina State

University, USA

\section{${ }^{*}$ Correspondence:}

Debra Mohnen, Department of

Biochemistry and Molecular Biology,

Complex Carbohydrate Research

Center, University of Georgia, 315

Riverbend Rd., Athens,

GA 30602, USA

e-mail:dmohnen@ccrc.uga.edu
GAlactUronosylTransferase12 (GAUT12)/IRregular Xylem8 (IRX8) is a putative glycosyltransferase involved in Arabidopsis secondary cell wall biosynthesis. Previous work showed that Arabidopsis irregular xylem8 (irx8) mutants have collapsed xylem due to a reduction in xylan and a lesser reduction in a subfraction of homogalacturonan $(H G)$. We now show that male sterility in the irx8 mutant is due to indehiscent anthers caused by reduced deposition of xylan and lignin in the endothecium cell layer. The reduced lignin content was demonstrated by histochemical lignin staining and pyrolysis Molecular Beam Mass Spectrometry (pyMBMS) and is associated with reduced lignin biosynthesis in irx8 stems. Examination of sequential chemical extracts of stem walls using 2D ${ }^{13} \mathrm{C}^{-1} \mathrm{H}$ Heteronuclear Single-Quantum Correlation (HSOC) NMR spectroscopy and antibody-based glycome profiling revealed a reduction in $\mathrm{G}$ lignin in the $1 \mathrm{M} \mathrm{KOH}$ extract and a concomitant loss of xylan, arabinogalactan and pectin epitopes in the ammonium oxalate, sodium carbonate, and $1 \mathrm{M} \mathrm{KOH}$ extracts from the irx 8 walls compared with wild-type walls. Immunolabeling of stem sections using the monoclonal antibody CCRC-M138 reactive against an unsubstituted xylopentaose epitope revealed a bi-lamellate pattern in wild-type fiber cells and a collapsed bi-layer in irx8 cells, suggesting that at least in fiber cells, GAUT12 participates in the synthesis of a specific layer or type of xylan or helps to provide an architecture framework required for the native xylan deposition pattern. The results support the hypothesis that GAUT12 functions in the synthesis of a structure required for xylan and lignin deposition during secondary cell wall formation.

Keywords: secondary cell walls, xylan, lignin, pectin, wall glycan epitopes, anther dehiscence

\section{INTRODUCTION}

The plant extracellular matrix (i.e., cell wall) consists of a variety of complex carbohydrate polymers with distinct chemical and physical properties. The covalent and noncovalent interactions between these polymers in the final composite determine many of the characteristics of the cell wall. Accordingly, mutations in individual glycosyltransferases (GTs), each of which presumably participates in the biogenesis of a single cell wall component or domain, often affect multiple classes of cell wall polymers and sometimes result in dwarf plants. For example, the Arabidopsis mutant $\operatorname{irx} 7$ (Zhong et al., 2005) is defective in both xylan and cellulose deposition, whereas qua1 (Bouton et al., 2002; Leboeuf et al., 2005; Orfila et al., 2005), parvus-3/gatl1 (Lao et al., 2003; Shao et al., 2004; Brown et al., 2007; Lee et al., 2007b; Kong et al.,
2009), and irx8/gaut12 mutants (Peña et al., 2007; Persson et al., 2007) are affected in pectin and xylan biosynthesis. These complex effects make it difficult to infer primary gene function on the basis of mutant phenotypes alone.

The gene mutated in the xylan- and pectin-deficient mutant irregular xylem8 (irx8) is GAUT12/IRX8 (At5g54690), a member of CAZy family GT8 that contains GTs related to homogalacturonan (HG): $\alpha$-1,4-galacturonosyltransferase (GalAT) GAUT1 (Sterling et al., 2006). In this paper we refer to the gene affected in the irx8 mutant as GAUT12 and its protein as GAlactUronosylTransferase12 (GAUT12). GAUT12 is predicted to be a type II transmembrane protein with its C-terminal catalytic domain facing the Golgi lumen. Transient expression of YFP-tagged GAUT12 protein showed that it co-localizes with 
CFP-tagged MUR4, consistent with the localization of GAUT12 in the Golgi apparatus (Peña et al., 2007). Transcription of GAUT12 is strongest in xylem vessels and interfascicular fiber cells, and irx8 mutant cell walls show a substantial reduction in glucuronoxylan (Peña et al., 2007; Persson et al., 2007) as well as a modest reduction in $\alpha$-1,4-linked GalA (Persson et al., 2007). Xylan is one of the major components of the secondary wall, and pectin is a major matrix polysaccharide in primary walls, but is also found in low abundance in walls prepared from cells synthesizing secondary walls. Additionally, irx 8 mutant xylan is nearly devoid of a xylan reducing-end glycosyl sequence [XRES; $\beta$-D-Xyl $p-(1 \rightarrow 3)$ $\alpha$-L-Rhap- $(1 \rightarrow 2)-\alpha$-D-GalpA- $(1 \rightarrow 4)$-D-Xylp] (Peña et al., 2007). XRES has been proposed to act either as a primer to initiate xylan biosynthesis or a terminator to control xylan chain length (York and O'Neill, 2008). However, it remains unknown how XRES synthesis is initiated. Since stem microsomes isolated from irx 8 plants contain comparable amounts of xylan:xylosyltransferase and xylan:glucuronosyltransferase activity as their wild-type counterparts (Brown et al., 2007; Lee et al., 2007a), it seems unlikely that GAUT12 is involved in the elongation or branching of the xylan backbone (York and O'Neill, 2008; Scheller and Ulvskov, 2010). Based on analyses of irx 8 cell walls and GAUT12 protein homology to GAUT1, it has been hypothesized that GAUT12 is a GalAT that either synthesizes a subfraction of HG (Persson et al., 2007) or catalyzes the addition of GalA into the nascent XRES (Peña et al., 2007). The biochemical function of GAUT12, however, remains unresolved to date.

In addition to being severely dwarfed and slow growing, Arabidopsis irx8 mutants are sterile (Persson et al., 2007). Consistent with a role in secondary wall formation and reproduction, GAUT12 expression is regulated by transcription factors that regulate vessel and fiber formation, such as MYB46 (Ko et al., 2009), MYB83 (McCarthy et al., 2009), VND6, and VND7 (Yamaguchi et al., 2010), as well as by transcription factors that act in anther development, such as MYB26/MALE STERILE35 (Steiner-Lange et al., 2003; Yang et al., 2007), NST1/NST2 (Mitsuda et al., 2005), and AHP4 (Jung et al., 2008). Within anthers, secondary wall thickenings in the endothelium cell layer provide part of the biophysical force that enables dehiscence, the programmed rupture of the anthers to release mature pollen (Wilson et al., 2011). Several lignin-defective mutants have recently been shown to be indehiscent and to generate defective pollen grains (Schilmiller et al., 2009; Weng et al., 2010; Thevenin et al., 2011).

The phenotypes of the Arabidopsis irx 8 mutant include not only a loss of $\sim 60 \%$ xylan and $\sim 13 \%$ pectin, but also $\sim 25 \%$ cellulose. This is a significantly smaller reduction in cellulose than observed in the cellulose defective mutants irx1 and irx3 (Brown et al., 2005; Persson et al., 2005). Based on phloroglucinol-HCl staining, a reduction of lignin in irx 8 vessels and fiber cells was also recently reported that was suggested to be associated with reduced xylan biosynthesis (Petersen et al., 2012). Lignin, a resinlike molecular network generated by oxidative polymerization of phenolic subunits within the extracellular space of many terminally differentiated cells constitutes up to $30 \%$ of most secondary cell walls (Boerjan et al., 2003). The three subunits of lignin, namely $p$-hydroxyphenyl $(\mathrm{H})$, guaiacyl $(\mathrm{G})$, and syringyl (S) are laid down in specific spatio-temporal patterns. For example, in wood formation $\mathrm{H}$ and $\mathrm{G}$ lignins are deposited at early stages of lignification in the middle lamella and tricellular junctions, while $\mathrm{G}$ lignin is deposited earlier than $\mathrm{S}$ lignin in vessels and fibers and S lignin is mainly deposited in fibers (Donaldson, 2001). Lignin subunits also become covalently linked to hemicelluloses and pectins (Jeffries, 1990), and the carbohydrate polymers are thought to guide expansion of the lignin lamellae during lignification (Donaldson, 2001; Donaldson and Knox, 2012).

The goal of this study was to elucidate the biological function(s) of GAUT12 in Arabidopsis. We identified the cause of sterility in the $\operatorname{irx} 8$ mutant and a reduction in lignin in this mutant. We attempted to solve the biochemical function of GAUT12 and found that GAUT12 does not have HG:GalAT activity comparable to that of GAUT1. In addition, an increased expression of an RG-I epitope in irx8 fiber cell walls was revealed. Our results suggest a connection between an RG-I-containing structure and the GAUT12-dependent wall product. We propose that GAUT12 participates in the synthesis of a structure required for xylan and lignin deposition during the formation of the secondary cell wall and that pectin is associated with this structure.

\section{MATERIALS AND METHODS PLANT MATERIALS}

Arabidopsis wild type (Col-0), irx8-5 (SALK_044387), irx8-2 (SAIL_603_G02), parvus-3 (SALK_045368), and irx9-1 (SALK_058238) plants were grown on soil in a controlledenvironment chamber (Conviron, Pembina, ND) under a 14-h-light/10-h-dark cycle at 19 and $15^{\circ} \mathrm{C}$, respectively. Light intensity was $150 \mu \mathrm{Em}^{-2} \mathrm{~s}^{-1}$ and relative humidity was maintained at 50\%. Plants were harvested after 7-8 weeks. T-DNA insertions were confirmed using primers from genomic regions flanking the T-DNA and the general T-DNA left border primer (Supplemental Table S1). Arabidopsis Col-0 and irx8 heterozygote plants were transformed via the floral dip method (Clough and Bent, 1998) and transgenic plants selected on $1 / 2$ MS media plates containing $15 \mathrm{mg} / \mathrm{L}$ hygromycin. Transgenic plants harboring the construct in Col-0 and irx 8 homozygote mutant backgrounds were genotyped using PCR (Primers listed in Supplemental Table S1).

\section{GENERATION OF THE GAUT12-EGFP CONSTRUCT}

GAUT12 coding sequence (CDS) was amplified from total RNA $(0.5 \mu \mathrm{g})$ isolated from 7-week-old Arabidopsis Col-0 stem by RT-PCR using the SuperScriptTM III One-Step RT-PCR System with Platinum Tag High Fidelity (Invitrogen 12574-030) and cloned into pGEM $^{\circledR}-\mathrm{T}$ Easy vector (Promega) (primers listed in Supplemental Table S1). The amplified GAUT12 CDS was sequence-verified and cloned into the over-expression construct pCambia35tl:egfps2\#4 (Pattathil et al., 2005) between the NcoI and KpnI restriction sites to produce the GAUT12EGFP construct driven by the CaMV 35S promoter. The plasmid was electroporated into Agrobacterium tumefaciens strain GV3101 competent cells and the transformed cells used to transform both wild-type (WT) and heterozygous irx8 Arabidopsis plants. 


\section{HISTOCHEMICAL STAINING}

Mäule reagent was prepared as described (Chapple et al., 1992) with slight modifications and used to detect S lignin. Arabidopsis open flowers and hand-cut stem transverse sections were treated with $0.5 \%(\mathrm{w} / \mathrm{v}) \mathrm{KMnO}_{4}$ solution for $10 \mathrm{~min}$ and rinsed with water. For flower samples, the solution was supplemented with $0.01 \%(\mathrm{v} / \mathrm{v}) 7 \mathrm{X}$ detergent (Linbro, Flow Laboratories) to break surface tension. Samples were treated with $10 \%(\mathrm{v} / \mathrm{v}) \mathrm{HCl}$ for $5 \mathrm{~min}$, rinsed with water and mounted in concentrated ammonia for microscopic observation.

Phloroglucinol-HCl stain was prepared freshly as described (Guo et al., 2001). Two parts of $2 \%$ (w/v) phloroglucinol in 95\% (v/v) ethanol were mixed with one part concentrated $\mathrm{HCl}$. Pictures were taken $10 \mathrm{~min}$ after applying the stain. Stained flowers were viewed using a dissecting scope (Olympus SZH) under dark field. Stained stem transverse sections were viewed using a Nikon Eclipse80i microscope under bright field. Images were captured using a Nikon DS-Ril camera head (Nikon, Melville, NY).

\section{SCANNING ELECTRON MICROSCOPY}

Using a dissecting scope, anthers from WT and mutant open flowers were removed with dissecting forceps (Sigma-Aldrich T4537). Pollen was released onto specimen stubs topped with doublesided sticky carbon tabs by gently tapping the forceps, or lightly tapping the anthers onto the stubs. The irx 8 mutant anthers were first manually dissected to open them and pollen was gently scooped out using the forceps tip and transferred onto the stub surface. Samples were dehydrated and coated with gold particles for $120 \mathrm{~s}$ in a Sputter Coater, and imaged using either a JEOL JSM5800 (SEM/EDAX) scanning electron microscope or a Topcon Aquila-Hybrid SEM.

\section{IN VITRO POLLEN TUBE GROWTH AND RNA PREPARATION}

Pollen tubes were grown in vitro as previously described (Dardelle et al., 2010). In brief, WT pollen grains were collected from 40 open flowers by vortexing for $3 \mathrm{~min}$ in a microcentrifuge tube containing $1 \mathrm{ml}$ pollen germination medium (PGM) composed of $5 \mathrm{mM} \mathrm{CaCl} \cdot 2 \mathrm{H}_{2} \mathrm{O}, 0.01 \%$ (w/v) $\mathrm{H}_{3} \mathrm{BO}_{3}$, $5 \mathrm{mM} \mathrm{KCl}, 1 \mathrm{mM} \mathrm{MgSO}_{4} \cdot 7 \mathrm{H}_{2} \mathrm{O}$, and $10 \%$ (w/v) sucrose ( $\mathrm{pH}$ adjusted to 7.5 using $\mathrm{KOH}$ ). The flowers were carefully removed and pollen grains pelleted by $3200 \mathrm{~g}$ centrifugation for $6 \mathrm{~min}$. The old media was removed, the pollen pellet gently re-suspended in $250 \mu \mathrm{L}$ of fresh (PGM), and the pollen grains transferred into a $13 \times 100 \mathrm{~mm}$ glass tube, covered with $3 \mathrm{M}$ micropore tape and set in the dark at $22^{\circ} \mathrm{C}$ for 6 or $24 \mathrm{~h}$.

For RNA isolation, pollen grains from 200 open WT flowers were collected in PGM in five tubes. Pollen from 200 flowers was either directly harvested as hydrated pollen grains $(0.5 \mathrm{~h})$ or grown as pollen tubes for 6 and $24 \mathrm{~h}$. Hydrated pollen grains $(0.5 \mathrm{~h})$ were combined and ground in liquid nitrogen using a plastic pestle and a microcentrifuge tube. Pollen tubes grown for $6 \mathrm{~h}$ and $24 \mathrm{~h}$ for RNA isolation were collected by centrifugation for $6 \mathrm{~min}$ at $3200 \mathrm{~g}$ and ground in microcentrifuge tubes. RNA isolation was repeated using three batches of independently collected tissues.

\section{TISSUE FIXATION AND IMMUNOLABELING}

Freshly cut plant tissues were fixed in $25 \mathrm{mM}$ sodium phosphate buffer ( $\mathrm{pH} 7.1$ ) with $1.6 \%(\mathrm{w} / \mathrm{v})$ paraformaldehyde and $0.2 \%$ $(\mathrm{w} / \mathrm{v})$ glutaraldehyde overnight at $4^{\circ} \mathrm{C}$. Using a lab-grade microwave (PELCO BioWave Pro, Ted Pella, CA) set at 250 Watt, tissues were washed three times for $1 \mathrm{~min}$ each with $25 \mathrm{mM}$ sodium phosphate buffer ( $\mathrm{pH}$ 7.1) followed by two washes with water. Samples then underwent a series of 40s ethanol gradient incubations $(35,50,75,95,100,100$, and $100 \%[\mathrm{v} / \mathrm{v}])$ to dehydrate the tissue. Samples were infiltrated with cold LR White embedding resin (Ted Pella) in a gradient $(1: 3,1: 1,3: 1$ resin:ethanol $[\mathrm{v} / \mathrm{v}])$ and finally three times with $100 \%$ resin. Each step was conducted under vacuum $\left(20^{\prime \prime} \mathrm{Hg}\right)$ for $2.5 \mathrm{~min}$. After the last resin change, samples were kept at $4^{\circ} \mathrm{C}$ for $24 \mathrm{~h}$, transferred into gelatin capsules filled with resin, and polymerized under $365 \mathrm{~nm}$ UV light at $4^{\circ} \mathrm{C}$ for $48 \mathrm{~h}$. Tissue cross sections $(250 \mathrm{~nm})$ were cut with a Leica EM UC6 ultramicrotome (Leica Microsystems), mounted on pre-coated slides (Colorfrost/Plus, Fisher Scientific) and used for immunolabeling or stained with $0.05 \%(\mathrm{w} / \mathrm{v})$ toluidine blue for light microscopy.

Immunolabeling for fluorescent microscopy was done as described (Avci et al., 2012). For LM series and JIM series antibodies, a wash buffer containing $10 \mathrm{mM} \mathrm{KPBS}$ ( $\mathrm{pH} 7.2$ ) and $100 \mathrm{mM} \mathrm{NaCl}$ was used because we found that the use of $500 \mathrm{mM}$ $\mathrm{NaCl}$ adversely affected consistent binding of these antibodies. The secondary antibody used for the LM and JIM series was Alexa fluor 488 goat anti-rat IgG (Cat\#A11006, Invitrogen) which was applied to the sections in the same manner as described above, but diluted in the low salt wash buffer. Images were captured using a Nikon DS-Ril camera head (Nikon, Melville, NY). All data shown depict representative images out of three images viewed for each type of tissue section stained with each antibody.

\section{TRANSMISSION ELECTRON MICROSCOPY}

Ultrathin sections $(80 \mathrm{~nm})$ were prepared using an ultramicrotome (Leica EM UC6, Austria) and collected on Formvarcoated nickel grids. Grids were stained with $2 \%(\mathrm{w} / \mathrm{v})$ uranyl acetate for $4 \mathrm{~min}$ followed by 10 dips in three changes of deionized water and dried by wicking. Micrographs were recorded on film in a JEOL $100 \mathrm{~S}$ transmission electron microscope. The negatives were developed and scanned in Adobe Photoshop.

For immunogold labeling, ultrathin sections were blocked in TBS (10 mM Tris buffer, $150 \mathrm{mM} \mathrm{NaCl}, \mathrm{pH} 7.5)$ containing $0.06 \%(\mathrm{w} / \mathrm{v})$ bovine serum albumin for $30 \mathrm{~min}$ at room temperature in a petri-dish with a folded piece of water-soaked Kimwipe set to one side of the dish. Sections were transferred onto $10 \mu \mathrm{L}$ drops of primary antibody diluted (1:5) in TBS for $1 \mathrm{~h}$. After washing three times by dipping the grids (10 times) in TBS, secondary antibody (goat anti-rat IgG coupled to $15 \mathrm{~nm}$ gold,) diluted $1: 10$ in TB (10 mM Tris buffer) containing $0.06 \%(\mathrm{w} / \mathrm{v})$ bovine serum albumin was applied to the sections. The sections were dipped in TB and distilled water for washing and dried by wicking. Images shown are representative of four images viewed for each sample stained with each antibody. 


\section{STEM RNA ISOLATION AND QUANTITATIVE PCR ANALYSES}

The bottom half of stem tissues from wild type and irx8, in which secondary cell walls are actively synthesized, were flash frozen in liquid nitrogen and ground to a fine powder in liquid nitrogen in pre-chilled mortars with pestles. Total RNA was isolated from $\sim 100 \mathrm{mg}$ of frozen powder from three individual tissue samples using RNeasy Plant Mini Kit (Qiagen, 74904). First-strand cDNA was synthesized from $1 \mu \mathrm{g}$ of total RNA using SuperSript III FirstStrand Synthesis Super mix (Invitrogen, 18080-400) followed by quantitative PCR analysis using $\mathrm{iQ}^{\mathrm{TM}}$ SYBR Green Supermix (BioRad 170-8882) on a CFX96 ${ }^{\mathrm{TM}}$ Real-Time PCR Detection System (Bio-Rad) following the manufacturer's instructions. Melt curve analyses were performed after each run to ensure single size amplicon production. Data are the average \pm standard deviation of three biological samples. The data were analyzed as described (Livak and Schmittgen, 2001). Primer sequences are provided in Supplemental Table S1.

\section{CELL WALL (AIR) PREPARATION}

Whole stem tissues were harvested from 7-week-old Col-0, irx85, irx8-5+GAUT12, irx8-2+GAUT12, and WT+GAUT12, ground with a mortar and pestle to a fine powder in liquid nitrogen, re-suspended in $80 \%(\mathrm{v} / \mathrm{v})$ ethanol and rotated end-to-end for $12 \mathrm{~h}$. The pellet obtained upon $4000 \mathrm{rpm}$ centrifugation was sequentially washed in $80 \%(\mathrm{v} / \mathrm{v})$ ethanol, $100 \%$ ethanol, chloroform: methanol $(1: 1, \mathrm{v} / \mathrm{v})$, and acetone by re-suspension, rotation for $12 \mathrm{~h}$, and re-centrifugation. The resulting alcohol insoluble residue (AIR) was dried for $24 \mathrm{~h}$ at room temperature.

\section{GLYCOSYL RESIDUE COMPOSITION ANALYSES}

Neutral sugar analysis was performed as described (Albersheim et al., 1967) with slight modifications. Each AIR sample $(0.4 \mathrm{mg}$ with $20 \mu \mathrm{g}$ myo-inositol as an internal standard) was hydrolyzed with 30 drops of $2 \mathrm{~N}$ trifluoroacetic acid (TFA) for $2 \mathrm{~h}$ at $120^{\circ} \mathrm{C}$. Samples were cooled to room temperature and dried under an air stream, washed twice with isopropanol to remove TFA, and reduced by incubation for $1 \mathrm{~h}$ at room temperature in 10 drops of sodium borohydride $(10 \mathrm{mg} / \mathrm{ml})$ dissolved in $1 \mathrm{M}$ ammonium hydroxide solution. The reaction was quenched with 30-40 drops of acetone and dried down with air. Once the volume was reduced to half, isopropanol $(1 \mathrm{ml})$ was added to each sample to facilitate drying. $O$-acetylation was performed by adding $250 \mu \mathrm{l}$ of acetic anhydride followed by $230 \mu \mathrm{l}$ of concentrated TFA and the samples were incubated for $10 \mathrm{~min}$ at $50^{\circ} \mathrm{C}$. Samples were then washed with isopropanol and dried down with air. $\mathrm{H}_{2} \mathrm{O}$ $(1 \mathrm{ml})$ and dichloromethane (DCM, $1 \mathrm{ml})$ were added and the samples vortexed and centrifuged to allow phase separation. The top aqueous layer was discarded and the bottom DCM layer containing the alditol acetate derivatives was dried down. Ten drops of DCM were added to each sample before analysis by gas-liquid chromatography-flame ionization detection using an Agilent 7890A GC system.

For GalA and GlcA measurements, AIR samples (0.5 mg) were hydrolyzed in $2 \mathrm{~N}$ TFA at $105^{\circ} \mathrm{C}$ for $1 \mathrm{~h}$. The hydrolysates were dried down with an air stream, re-hydrolyzed in $3 \mathrm{~N}$ methanolic HCl (Thermo Scientific, Rockford, IL) overnight at $80^{\circ} \mathrm{C}$, dried down individually and dissolved in $100 \mu$ l distilled
$\mathrm{H}_{2} \mathrm{O}$. The samples were centrifuged and $12.5 \mu \mathrm{l}$ of hydolysate supernatant was loaded onto a CarboPac PA20 analytical column $(3 \times 150 \mathrm{~mm}$, Dionex, Sunnyvale, CA). The loaded column was washed with a solution of $49 \mathrm{mM} \mathrm{NaOH}, 20 \mathrm{mM} \mathrm{NaOAc}$ and eluted with a linear gradient from $49 \mathrm{mM} \mathrm{NaOH}, 20 \mathrm{mM}$ $\mathrm{NaOAc}$ to $40 \mathrm{mM} \mathrm{NaOH}, 200 \mathrm{mM} \mathrm{NaOAc}$ over $25 \mathrm{~min}$. The column was eluted at $30^{\circ} \mathrm{C}$ and a flow rate of $0.4 \mathrm{ml} / \mathrm{min}$ and effluent was monitored with an ECD detector. The amount of GalA and GlcA was determined by comparison of peak areas to standards separated under the same conditions.

Each analysis was repeated five times for each sample, and the bar represents the average mol\% of each sugar residue \pm standard error.

\section{SEOUENTIAL EXTRACTION AND GLYCOME PROFILING}

Sequential extractions of cell wall samples and glycome profiling were carried out as described previously (Demartini et al., 2011; Pattathil et al., 2012). Briefly, AIR samples were sequentially extracted with $50 \mathrm{mM}$ ammonium oxalate, $\mathrm{pH} 5 ; 50 \mathrm{mM}$ sodium carbonate [containing $0.5 \%(\mathrm{w} / \mathrm{v})$ sodium borohydride], $\mathrm{pH} 10$; $1 \mathrm{M} \mathrm{KOH}$ with $1 \%(\mathrm{w} / \mathrm{v})$ sodium borohydride; $4 \mathrm{M} \mathrm{KOH}$ with $1 \%$ (w/v) sodium borohydride; $100 \mathrm{mM}$ acidified sodium chlorite; and finally with $4 \mathrm{M} \mathrm{KOH}$ with $1 \%$ (w/v) sodium borohydride for the post-chlorite extraction. The wall extracts were used for NMR analyses (see below) and were screened by ELISA using plant glycan-directed monoclonal antibodies (CCRC, JIM, and MAC series) from Complex Carbohydrate Research Center stocks available through CarboSource Services (http://www.carbosource. net). Detailed description of each mAbs used in this study can be found in the Supporting Information (Supplemental Table S2) that includes links to a web database named WallMAbDB (http:// www.wallmabdb.net).

\section{PYROLYSIS MOLECULAR BEAM MASS SPECTROMETRY (pyMBMS)}

About $4 \mathrm{mg}$ of sample was weighed and transferred into 80$\mu l$ stainless steel sample cups of an auto sampler of a double shot pyrolyzer (PY-2020iD, Frontier Ltd.). The samples were pyrolyzed at $500^{\circ} \mathrm{C}$ and the residues analyzed using a custom built Super Sonic Molecular Beam Mass Spectrometer (Extrel Model MAX-1000).

Mass spectral data from m/z 30-450 were acquired on a Merlin Automation Data System version 3.3. Multivariate analysis was performed using Unscrambler software version 10.1 (CAMO). The intensities of the lignin peaks were summed and averaged to estimate the lignin content in the sample (Evans and Milne, 1987). Total lignin peaks corresponded to $\mathrm{m} / \mathrm{z} 120,124,137,138$, $150,152,154,164,167,168,178,180,181,182,194,208$, and 210 . The syringyl peaks corresponded to $\mathrm{m} / \mathrm{z} 154,167,168,182$, 194,208 , and 210, the guaiacol peaks corresponded to $\mathrm{m} / \mathrm{z} 124$, $137,138,150,164$, and 178, and phenol peaks corresponded to $\mathrm{m} / \mathrm{z} 120$ and 122. Syringyl to Guaiacol (S/G) ratios were determined by summing syringyl peaks and dividing by the sum of guaiacol peaks. The lignin values thus generated and calculated were compared with the WT. Because the NIST (National Institute Standards and Technology) standard for Arabidopsis is not available, the lignin percentage was corrected using a standard of eastern cottonwood (NIST 8492) to obtain the lignin 
percentage value equivalent to the Klason lignin. This analysis was conducted at the Complex Carbohydrate Research Center Analytical Services, University of Georgia (http://www.ccrc.uga. edu/services/ccrcanalyticalservices/index.html).

\section{DETERMINATION OF LIGNIN MONOMER COMPOSITION BY HSOC NMR SPECTROSCOPY}

Perdeuterated pyridinium molten salt (ionic liquid) was synthesized as described (Jiang et al., 2009). Approximately $2 \mathrm{mg}$ of cell wall extracts were weighed and dissolved in $180 \mu \mathrm{l}$ of the ionic liquid [DMSO- $d_{6} /$ pyridine- $\left.d_{5}(2: 1, \mathrm{v} / \mathrm{v})\right]$ at $65^{\circ} \mathrm{C}$. Data were collected at $60^{\circ} \mathrm{C}$ on Agilent $600 \mathrm{MHz}$ Direct Drive spectrometers equipped with either a 5 or $3 \mathrm{~mm}$ cold probe. A standard Agilent pulse program ("HSQCAD") was used to acquire the ${ }^{13} \mathrm{C}$ ${ }^{1} \mathrm{H}$ heteronuclear correlated spectra. The proton dimension of 1200 complex data points covered $20 \mathrm{ppm}$ centered at $6 \mathrm{ppm}$, and the carbon dimension of 48 or 64 complex points was centered at $92 \mathrm{ppm}$ with a width of either 100 or $184 \mathrm{ppm}$, respectively. In the former case, some resonances in the alkyl or aromatic regions were folded along the $\mathrm{F} 1$ axis. Total data acquisition times ranged from 7 to $16 \mathrm{~h}$, with the number of transients between 240 and 400 per $t_{1}$ increment. Data were processed with NMRPipe (NIH) and visualized with NMRViewJ (One Moon Scientific) or with MNova (Mestrelab Research). Typically, squared cosine window functions were applied in both dimensions after zero filling and linear prediction in $t_{1}$. Chemical shifts were referenced to DMSO at $2.50 \mathrm{ppm}$ in proton and $39.51 \mathrm{ppm}$ in carbon. Heteronuclear Single-Quantum Correlation (HSQC) cross-peak assignments were determined and referenced as described (Kim and Ralph, 2010).

\section{GENERATION OF ARABIDOPSIS SUSPENSION CULTURES AND GAUT12-EGFP TRANSGENIC CELLS}

A wild-type Arabidopsis suspension culture was generated from seed callus as previously described (Doelling and Pikaard, 1993). Cell were subcultured every 10 days by transfer of $7 \mathrm{ml}$ of packed cells into $100 \mathrm{ml}$ fresh callus inducing medium (CIM). CIM contained $3.2 \mathrm{~g} / \mathrm{L}$ Gamborg's B-5 basal medium with minimal organics (Sigma-Aldrich G5893 or PhytoTechnology Laboratories G398), $2 \mathrm{mg} / \mathrm{L}$ 2,4-D, $0.05 \mathrm{mg} / \mathrm{L}$ kinetin, and $20 \mathrm{~g} / \mathrm{L}$ sucrose $(\mathrm{pH}$ 5.7). The GAUT12-EGFP construct was stably transformed into wild-type suspension culture cells via Agrobacterium tumefaciens strain GV3101. An aliquot of $1 \mathrm{ml}$ packed cells from a four-dayold culture was co-cultured in $8 \mathrm{ml}$ of fresh CIM with $100 \mu \mathrm{l}$ of Agrobacterium cells harboring the GAUT12-EGFP vector resuspended in CIM to an $\mathrm{OD}_{600}$ of 0.8 . The Agrobacteria were previously seed-cultured overnight in $3 \mathrm{ml}$ YEP medium supplemented with rifampicin $50 \mathrm{mg} / \mathrm{L}$, kanamycin $50 \mathrm{mg} / \mathrm{L}$, and gentamycin $50 \mathrm{mg} / \mathrm{L}$ and re-cultured in $25 \mathrm{ml}$ of the above YEP medium. The co-culture was done in a 1-inch-deep petri-dish in the dark on a gyrotory shaker at $130 \mathrm{rpm}$ for $24 \mathrm{~h}$. Cells were removed from the co-cultivation medium and washed thrice with CIM and then with CIM supplemented with $500 \mathrm{mg} / \mathrm{L}$ cefotaxime in $50 \mathrm{ml}$ falcon tubes. In each wash, the old medium was completely removed and $20 \mathrm{ml}$ fresh medium introduced and vortexed for $30 \mathrm{~s}$. The washed cells were plated onto CIM $0.6 \%$ $(\mathrm{w} / \mathrm{v})$ agar plates containing $300 \mathrm{mg} / \mathrm{L}$ cefotaxime and $15 \mathrm{mg} / \mathrm{L}$ hygromycin. Hygromycin-resistant transgenic calli emerged in 2-3 weeks and were transferred onto fresh media plates and grown for up to 4 weeks. After two to three transfer cycles, the calli were free of Agrobacteria and used to initiate suspension cultures. Surviving calli were genotyped, and RT-PCR was used to determine the expression level of the GAUT12-EGFP transcript. The GAUT12-EGFP transcript was highest on day 5 compared to day 2 and 8 . Therefore, cells were harvested on day 6 for preparation of microsomes.

\section{MICROSOMAL MEMBRANE PREPARATION}

Microsomal membranes used for enzyme activity assays were prepared at $4^{\circ} \mathrm{C}$ as described (Orfila et al., 2005) with modification. Arabidopsis stems (10 grams) were cut into small pieces, flash frozen in liquid nitrogen, and homogenized on ice in $20 \mathrm{ml}$ of pre-chilled homogenization buffer containing $50 \mathrm{mM}$ Hepes (pH 7.3), 0.4 M sucrose, $0.1 \mathrm{M}$ sodium ascorbate, $0.25 \mathrm{mM}$ $\mathrm{MnCl}_{2}, 25 \mathrm{mM} \mathrm{KCl}, 1 \%$ (w/v) polyvinylpyrrolidone (PVPP), and EDTA-free protease inhibitor cocktail (Roche) until the tissues were pureed. The homogenate was filtered through three layers of miracloth and the filtrate centrifuged at $4^{\circ} \mathrm{C}$ for $30 \mathrm{~min}$ at $4000 \mathrm{~g}$ to remove cell debris and intact cells. The supernatant was ultra-centrifuged at $110,000 \mathrm{~g}$ for $1 \mathrm{~h}$, yielding the microsome pellet which was re-suspended on ice in pre-chilled storage buffer (homogenization buffer without PVPP, $30 \mu \mathrm{l}$ buffer/gram fresh weight) using a glass homogenizer. Total protein was measured using the Bradford assay (Bio-Rad Protein Assay 500-0006) with BSA as a standard. Aliquots of the microsomes were used directly or flash frozen in liquid nitrogen and stored at $-80^{\circ} \mathrm{C}$ for later use in enzyme assays and immunoprecipitation experiments.

\section{GENERATION OF anti-GAUT12 POLYCLONAL ANTIBODY AND WESTERN BLOTTING}

The anti-GAUT12 polyclonal antibody was generated against synthetic peptides corresponding to GAUT12 amino acid residues 101-114 (EQPLSEQELKGRSD) and antigen-purified over a column packed with the antigenic-peptide (service via New England Peptide, http://www.newenglandpeptide.com/). The purified anti-GAUT12 antibody did not cross-react with GAUT1 or GAUT7 (Supplemental Figure S11). Pre-immune serum did not contain anti-GAUT12 antibody. Anti-GAUT1 and antiGAUT7 antibodies were generated previously (Sterling et al., 2006; Atmodjo et al., 2011). For western blotting, a dilution factor of 1:5000, 1:10000, 1:3000, and 1:2000 was applied for anti-GAUT12, anti-GAUT7, anti-GAUT1, and anti-GFP (abcam, ab6556), respectively. Either horseradish peroxidase (HRP)- or alkaline phosphate (AP)-conjugated goat anti-rabbit secondary antibody (Sigma) was used followed by a reaction with corresponding substrates to yield a blue or purple color precipitant on the target protein band.

\section{IMMUNOPRECIPITATION OF GAUT12 FOR HG:GALAT ENZYME ASSAYS}

The purified anti-GAUT12 antibody $(1.14 \mathrm{mg} / \mathrm{ml})$ was incubated with Dynabeads M-280 Sheep anti-Rabbit IgG (Invitrogen, $112-04 \mathrm{D})$ in a ratio of $1: 20(\mathrm{v} / \mathrm{v})$ for $2 \mathrm{~h}$ at $4^{\circ} \mathrm{C}$ on a tube rotator and the beads collected on a magnet stand and washed thrice with isotonic PBS ( $\mathrm{pH} 7.4,139 \mathrm{mM} \mathrm{NaCl}, 5.5 \mathrm{mM} \mathrm{Na}_{2} \mathrm{HPO}_{4}, 1.2 \mathrm{mM}$ 
$\mathrm{NaH}_{2} \mathrm{PO}_{4}$ ). The beads were then washed once with storage buffer (see microsome preparation section). Each reaction contained $30 \mu \mathrm{l}$ anti-GAUT12-conjugated beads incubated with $\sim 500 \mu \mathrm{g}$ Triton X-100-treated microsomes at $4^{\circ} \mathrm{C}$ for $2 \mathrm{~h}$ on the tube rotator. The nonionic detergent Triton X-100 (TX-100) has been used to solubilize GalAT activity during GAUT1 and GAUT7 purification (Doong and Mohnen, 1998; Sterling et al., 2006; Atmodjo et al., 2011). A concentration of $4 \%(\mathrm{v} / \mathrm{v}) \mathrm{TX}-100$ was used to homogenize WT stem microsomes on ice and they were immediately diluted with storage buffer to a final detergent concentration of $0.5 \%(\mathrm{v} / \mathrm{v})$ for incubation with anti-GAUT12-conjugated magnetic beads.

After a $2 \mathrm{~h}$ end-to-end incubation at $4^{\circ} \mathrm{C}$, the beads were washed with pre-chilled storage buffer on ice thrice and the original $30 \mu \mathrm{l}$ beads were re-suspended in $13 \mu \mathrm{l}$ pre-chilled storage buffer for each enzyme reaction. Immunoabsorbed-GAUT1 was prepared by incubating Dynabeads M-280 Sheep anti-Rabbit IgG with anti-GAUT7 anti-serum in a ratio of $1: 3(\mathrm{v} / \mathrm{v})$ in parallel as described (Atmodjo et al., 2011). Anti-GAUT7 antibody was previously shown to immunoabsorb the HG:GalAT activitycontaining the GAUT1-GAUT7 core complex (Atmodjo et al., 2011).

\section{HG:GALAT ENZYME ACTIVITY ASSAY}

UDP-D- $\left[{ }^{14} \mathrm{C}\right] \mathrm{Gal} p \mathrm{~A} \quad$ (specific activity $180.3 \mathrm{mCi} / \mathrm{mmol}$; $1 \mathrm{Ci}=37 \mathrm{GBq})$ was synthesized enzymatically from UDPD- $\left[{ }^{14} \mathrm{C}\right]$ GlcpA using UDP-D-GlcpA 4-epimerase as described (Atmodjo et al., 2011). HG:GalAT activity was assayed in $30-\mu \mathrm{L}$ reactions containing either enzyme $(15 \mu \mathrm{l}$ total microsomes, $\sim 100 \mu \mathrm{g}$ total protein) or $13 \mu \mathrm{l}$ of immunoabsorbed beads, $50 \mathrm{mM}$ Hepes (pH 7.3), 0.2 M sucrose, 0.05\% (w/v) BSA, $25 \mathrm{mM}$ $\mathrm{KCl}, 1.9 \mathrm{mM} \mathrm{MnCl}_{2}, 1 \mathrm{mM} \mathrm{HG}$ oligosaccharides (referred to as oligogalacturonides, OGA) of a degree of polymerization (DP) of $7-23$, and $6.9 \mu \mathrm{M}$ UDP- $\left[{ }^{14} \mathrm{C}\right] \mathrm{Gal} p \mathrm{~A}$ (specific activity $180.3 \mathrm{mCi} / \mathrm{mmol} ; 1 \mathrm{Ci}=37 \mathrm{GBq}$ ). The reactions were incubated for $3 \mathrm{~h}$ at $29^{\circ} \mathrm{C}$ in a water-bath and terminated by the addition of $5 \mu \mathrm{l}$ of $400 \mathrm{mM} \mathrm{NaOH}$ with vortexing. The entire reaction $(\sim 35 \mu \mathrm{l})$ was spotted onto 1 -inch ${ }^{2}$ filter paper squares that had been pre-treated with cetylpyridinium chloride (CPC) as described (Sterling et al., 2005). The spotted filters were dried, washed $3 \times$ in $150 \mathrm{mM} \mathrm{NaCl}$ each for $15 \mathrm{~min}$ to remove access UDP- $\left[{ }^{14} \mathrm{C}\right] \mathrm{Gal} p \mathrm{~A}$, dried, and added to $4 \mathrm{ml}$ ScintiVerse ${ }^{\text {тм }} \mathrm{BD}$ cocktail for scintillation counting.

\section{LIQUID CHROMATOGRAPHY-TANDEM MASS SPECTROMETRY (LC-MS/MS)}

A large-scale GAUT12 immunoprecipitation was conducted for LC-MS/MS analysis. An aliquot of $500 \mu \mathrm{l}$ of the anti-GAUT12conjugated magnetic beads was incubated at $4{ }^{\circ} \mathrm{C}$ overnight on a tube rotator with WT Arabidopsis stem microsomes $(5 \mathrm{mg}$ total protein). The microsomes on ice were homogenized with $4 \%$ (v/v) TX-100 supplemented with $200 \mathrm{mM} \mathrm{NaCl}, 100 \mathrm{mM} \mathrm{NaOAc}$, and $2 \mathrm{mM}$ EDTA and immediately diluted with PBS to a final detergent concentration of $0.5 \%(\mathrm{v} / \mathrm{v})$ for incubation with antiGAUT12-conjugated magnetic beads. The beads were washed $5 \times$ with PBS $(\mathrm{pH} 7.4)$ and the recovered beads denatured in $3 \%(\mathrm{w} / \mathrm{v})$ SDS and reduced in $25 \mathrm{mM}$ DTT. The material bound to the beads was released by magnetic separation and separated by electrophoresis on a 10\% SDS-PAGE gel. A gel piece corresponding to the size of GAUT12 (between 55 and $70 \mathrm{KDa}$ protein marker) was cut out and in-gel trypsin-digested as described (Atmodjo et al., 2011). The peptide samples from the proteolytic digestions were analyzed on an Agilent 1100 capillary LC (Palo Alto, CA) interfaced directly to a LTQ linear ion trap mass spectrometer (Thermo Fisher, San Jose, CA). Mobile phases A and B were $\mathrm{H}_{2} \mathrm{O}-0.1 \%(\mathrm{v} / \mathrm{v})$ formic acid and acetonitrile- $0.1 \%(\mathrm{v} / \mathrm{v})$ formic acid, respectively. Peptides were eluted from the $\mathrm{C} 18 \mathrm{col}-$ umn into the mass spectrometer during an 80 min linear gradient from 5 to $55 \%(\mathrm{v} / \mathrm{v})$ mobile phase B at a flow rate of $4 \mu \mathrm{l} / \mathrm{min}$. The instrument was set to acquire MS/MS spectra on the nine most abundant precursor ions. Generated raw tandem mass spectra were converted into the mzXML format and then into peak lists using ReAdW software followed by mzMXL2Other software (Pedrioli et al., 2004). The peak lists were searched using Mascot 2.2 (Matrix Science, Boston, MA).

\section{DATABASE SEARCHING AND PROTEIN IDENTIFICATION}

A target database was created using the Arabidopsis annotated sequences obtained from the TAIR10_pep_20101214 protein database (ftp://ftp.arabidopsis.org/home/tair/Genes/TAIR10_ genome_release/TAIR10_blastsets / TAIR10_ pep_ 20101214 _ updated). A decoy database (decoy) was constructed by reversing the sequences in the normal database. Searches were performed against the normal and decoy databases using the following parameters: full tryptic enzymatic cleavage with two possible missed cleavages, peptide tolerance of $1000 \mathrm{ppm}$, fragment ion tolerance of $0.6 \mathrm{Da}$. Fixed modification was set as carbamidomethyl due to carboxyamidomethylation of cysteine residues $(+57 \mathrm{Da})$ and variable modifications were chosen as oxidation of methionine residues $(+16 \mathrm{Da})$ and deamidation of asparagine residues $(+1 \mathrm{Da})$. Statistically significant proteins from both searches were determined at a $\leq 1 \%$ protein false discovery rate (FDR) using the ProValT algorithm, as implemented in ProteoIQ (BioInquire, LLC, Athens, GA) (Weatherly et al., 2005).

\section{RESULTS}

\section{THE EFFECTS OF irX8 MUTATION ON GROWTH HABIT AND SECONDARY WALL SYNTHESIS IN XYLEM AND FIBERS ARE COMPLEMENTED BY A GAUT12-OVEREXPRESSION CONSTRUCT}

All reported irx8 mutant alleles are dwarf and show a collapsed xylem phenotype (Peña et al., 2007; Persson et al., 2007). We selected two of these GAUT12 T-DNA insertion mutants, irx8-2 and irx8-5, for this study (Supplemental Figure S1A). The T-DNA insertions in irx8-2 and irx8-5 are in the promoter region and fourth intron of GAUT12, respectively (Supplemental Figure S1A). Both alleles are associated with phenotypes similar to those previously described (Peña et al., 2007; Persson et al., 2007). Specifically, both mutants have a dwarfed growth habit (Supplemental Figure S1B), reduced secondary cell wall thickness in xylem and fiber cells, and collapsed xylem vessels (Supplemental Figures S1D,H). Semi-quantitative RT-PCR analyses revealed that irx8-2 plants contain trace amounts of full-length GAUT12 transcript, whereas irx8-5 plants contain transcripts that 
are truncated at the insertion site (Supplemental Figure S1L). Since both irx8-2 and irx8-5 mutants express similar overall phenotypes, they were used interchangeably in the present study.

The irx 8 mutant phenotypes in both alleles are complemented (Supplemental Figures S1E,I,K) by the constitutive expression of an EGFP-tagged GAUT12 construct (GAUT12-EGFP). The EGFP (Pattathil et al., 2005) was connected to the C-terminus of GAUT12 via a Val-Pro linker to facilitate structural flexibility between the two parts of the fusion protein and access of substrates to the predicted C-terminal catalytic domain of GAUT12 (Supplemental Figure S1A). The GAUT12-EGFP construct restored the phenotype of $\operatorname{irx} 8$ mutants as evidenced by the normal stature (Supplemental Figure S2B) and cell wall thickness (Supplemental Figures S3C,G) in the complemented transgenic plants. Cell wall sugar composition analyses revealed that irx8-5+GAUT12 and irx8-2+GAUT12 plants had a xylose content that was closer to normal (65 and $74 \mathrm{~mol} \%$ of wild-type level, respectively) compared to the irx8-5 mutant (33 mol\% of wild-type xylose content, Supplemental Figure S2C).
Finally, the 20 and $63 \mathrm{~mol} \%$ reduction of GalA and GlcA content, respectively, associated with the irx8-5 mutation was also complemented by the GAUT12-EGFP construct (Supplemental Figure S2D).

\section{REDUCTION IN LIGNIN AND XYLAN LEADS TO INDEHISCENT ANTHERS IN irx8}

Prior studies reported that multiple alleles of homozygous irx8 mutants were "semi-sterile" (Brown et al., 2005; Persson et al., 2007). Both the irx8-2 and irx8-5 mutants used for this study produced small and empty siliques with almost no seeds (Supplemental Figure S1M).

We investigated the cause of $\operatorname{ir} x 8$ sterility. First, we used a dissecting microscope to observe open flowers of ir $x 8$ and wild type (WT) at stages 13 and 14 (anthesis and fertilization) (Bowman, 1994), respectively (Figure 1). An open flower of irx8-5 contains reproductive organs of the correct shape but smaller in size than wild type (Figure 1A) and has shorter stamens (as previously described; Persson et al., 2007). At this stage, wild-type anthers
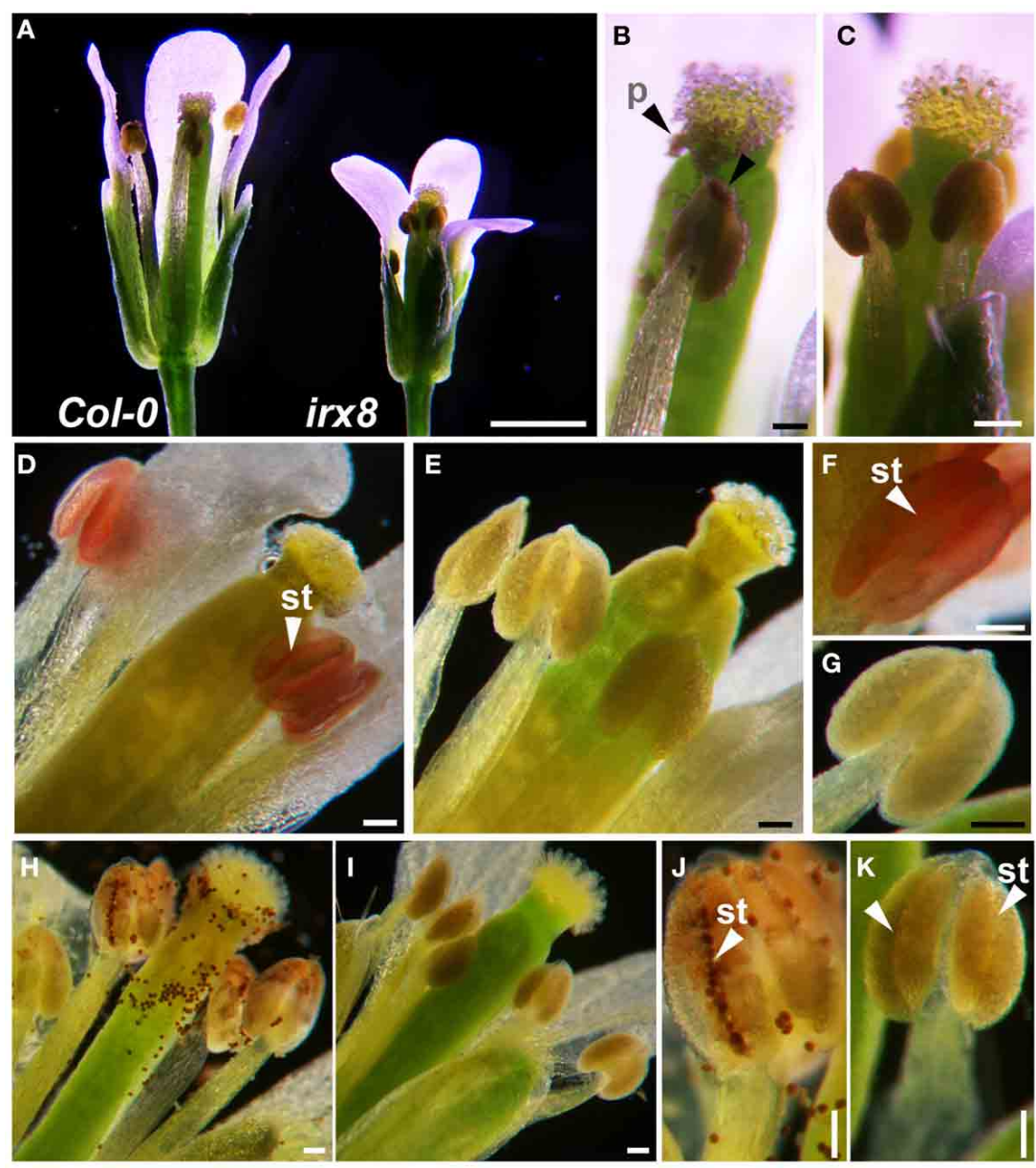

FIGURE 1 | Loss of lignin in anther endothecium cells results in failure of anther dehiscence in irx8 mutants. (A) Light microscope images of wild-type and irx 8 open flowers (stage 13 , anthesis). (B) Close-up of $\mathrm{CO}-0$ pistil and anthers. (C) Close-up of irx8-5 pistil and anthers. (D-G) Red phloroglucinol- $\mathrm{HCl}$ staining of flower anther indicates presence of lignin. Endothecium layer stains red in wild type (D), the lack of staining in irx8-5 (E), close-up of a wild-type anther (stomium indicated by arrow, F), and close-up of an irx8 anther showing no staining (G). (H-K) Mäule staining of flowers of wild type: pollen and dehisced anther walls stained red (H); lack of staining in irx8-5 (I); close-up of wild-type anther stomium (arrowhead), released pollen stained dark red $\mathbf{( J )}$; close-up of irx8-5 stomium (arrowhead), no lignin staining and no pollen released (K). Bar in $\mathbf{A}=1 \mathrm{~mm}$; bar in $\mathbf{B}-\mathbf{K}=0.1 \mathrm{~mm}$. p, pollen; st, stomium. 
have already dehisced and pollen grains are released onto the stigma and style (Figure 1B, arrowheads). The irx8-5 mutant, however, has smooth intact anthers and shows no pollen release (Figure 1C). The same phenotype was also observed in the irx8-2 mutant (Supplemental Figure S4).

Secondary wall thickening in endothecium cells of pollen sacs provides the mechanical force for anther dehiscence. After anther dehydration and pollen swelling, the stomium breaks open to release pollen at anthesis, followed by anther filament extension to achieve fertilization (Wilson et al., 2011). Phloroglucinol$\mathrm{HCl}$ staining (Wiesner test), which colorimetrically identifies coniferaldehyde end-groups in G lignin, and Mäule reagent, which reacts with syringylpropane moieties of S lignin to produce a rose red color (Lewis and Yamamoto, 1990) were used to compare lignification in the wild type and mutant. The lignin of the secondary wall thickening along the stomium furrow in wild-type mature anther stained red using phloroglucinol$\mathrm{HCl}$ (Figures 1D,F, arrowheads). In contrast, the stomium furrow was not stained at all in irx 8 anthers at the same stage (Figures 1E,G), suggesting a reduction of G lignin in the $i r x 8$ endothecium cell layer. In addition, released pollen from the wild type stained brownish-red with Mäule reagent at the stomium opening (Figure 1H). The wild-type pollen sacs were also partially stained since the stain was able to access the endothecium layer through the open stomium (Figure 1J). However, at the same developmental stage (anthesis), such staining was absent in irx 8 anthers (Figures 1I,K). Despite no pollen release in irx8 flowers, the anthers appeared to be turgid (Figure 1C; Supplemental Figure S4), suggesting that the pollen inside was swollen. The enzymatic lysis of the irx8 stomium and septum breakage also occurred in the anther, as seen in transverse sections (Figure 2E, black arrowheads). The indehiscent anther phenotype was confirmed in both the irx8-2 and irx8-5 mutants using SEM. This phenotype was complemented by the GAUT12-EGFP construct (Figure 3A; Supplemental Figure S4).

The secondary wall thickening of the endothecium cells (Figure 2A) contains not only lignin, but also xylan and cellulose (Wilson et al., 2011). Since irx 8 has a known xylan defect, we fixed, embedded, and sectioned open flowers of irx 8 and analyzed anther cell walls in the sectioned tissues using plant cell wall glycan-directed monoclonal antibodies against xylan and other major polysaccharides (Pattathil et al., 2010). In wildtype endothecium cell walls, LM10 (Figure 2B) which binds low-substituted xylan and LM11 (Figure 2C) which binds both low- and high-substituted xylan (McCartney et al., 2005) showed almost identical punctate labeling patterns, demonstrating a common location of the two xylan epitopes recognized by these two antibodies. Both epitopes are reduced in irx8 (Figures 2F,G),
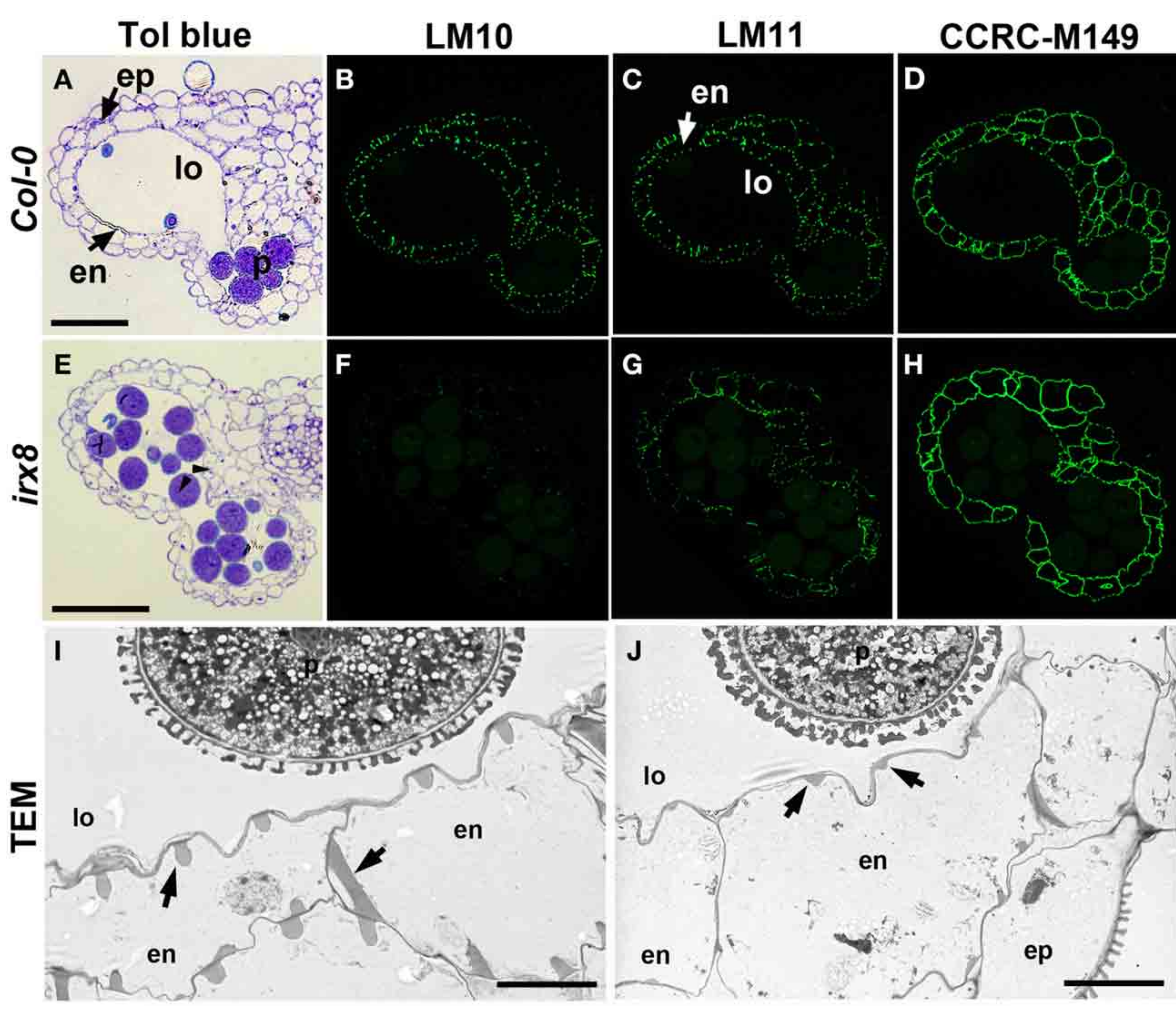

FIGURE 2 | Reduction in xylan deposition in anther endothecium cells in irx8 mutants. LR White-embedded WT (A) and irx8-5 (E) anther transverse sections $(250 \mathrm{~nm}$-thick) stained with toluidine blue O (Tol blue), arrowheads point to septum breakage. WT (B-D) and irx8 (F-H) anthers immunolabeled with xylan-reactive antibodies LM10
(B,F), LM11 (C,G), and CCRC-M149 (D,H). Bar = $50 \mu \mathrm{m}$ for $(\mathbf{A}-\mathbf{H})$ TEM of WT (I) and irx8-5 (J) anther. Arrows point to the secondary wall thickenings in the endothecium cell layer, which is reduced in irx8-5. ep, epidermis; en, endothecium; p, pollen; lo, locule. Bar $=5 \mu \mathrm{m}$ for (I) and (J). 


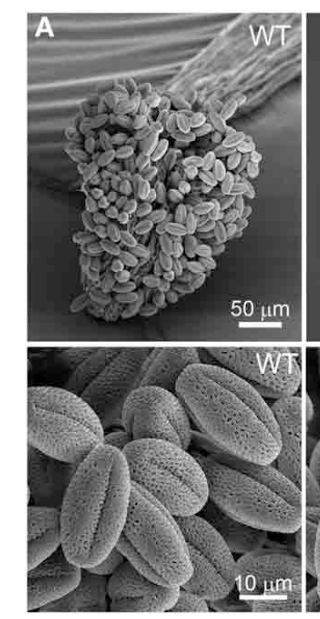

B

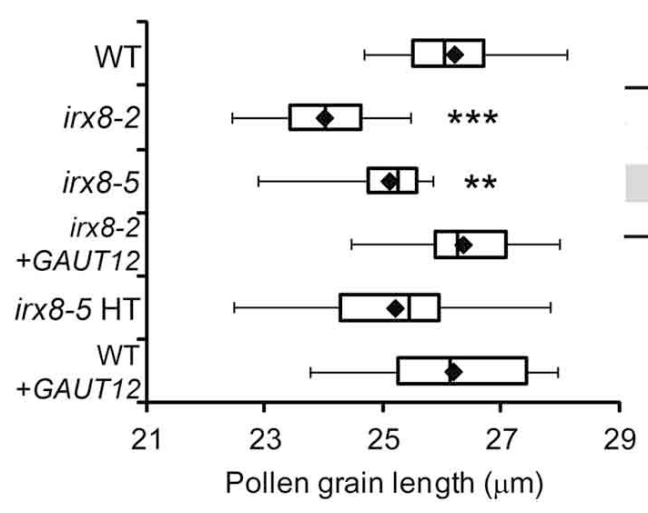

FIGURE 3 | SEM of anthers and pollen grains (PG) from irx8 and GAUT12-transgenic plants. (A) SEM images of anthers and PG from open flowers of WT, ir $x 8-2$, ir $x 8-5$, ir $x 8-2+$ GAUT12, ir $x 8-5$ heterozygote $(\mathrm{HT})$, and $\mathrm{WT}+$ GAUT12. Bar $=50 \mu \mathrm{m}$ in upper panel and $=10 \mu \mathrm{m}$ in lower panel. (B) Box plot diagram of PG length measured under SEM of WT $(n=10)$, ir $\times 8-2(n=19)$, ir $\times 8-5(n=21)$, ir $\times 8-2+\operatorname{GAUT12}(n=20)$, irx8-5 HT $(n=26)$, and WT+GAUT12 $(n=25)$. $n$, number of PG measured. The One-Way ANOVA was significant and a Bonferroni corrected post-hoc t-test indicates that PG length is significantly smaller in irx8-2 and ir $x 8-5$ and is complemented by GAUT12. The average pollen size of irx8 HT and WT+GAUT12 were similar to the size of WT pollen. $P_{t \text {-test }}<0.01\left(^{* *}\right)$, and $<0.001\left(^{* * *}\right)$. (C) The irx8 pollen viability test. Pollen from heterozygous irx8 plants was used to pollinate WT pistils and the progeny were genotyped by PCR. The accumulative $\chi^{2}$ value based on the total number of progeny was calculated for both alleles. Both were smaller than 3.84, which is the critical value for $\chi^{2}$ distribution at $\alpha=0.05$ and d.f. $=1$. The results showed that the HT vs. WT progeny fit the 1:1 ratio according to the Mendelian law of segregation. with LM10 labeling almost completely absent in irx8 (Figure 2F), indicating a pronounced reduction in low-substituted xylan recognized by LM10. Transmission electron microscopy (TEM) showed that the secondary wall thickening in wild-type endothecium cells, in the shape of teeth- and ribbon-like structures (Figure 2I, arrows), is significantly reduced in irx8 endothecium cells (Figure 2J, arrows). Xylan present in the irx8 endothecium was detected using CCRC-M149 (Figure 2H), CCRCM137, CCRC-M138, and CCRC-M160 antibodies (Supplemental Figures S5I,M,N), suggesting that GAUT12 affects some xylan synthesis in endothecium cells and that some xylan epitopes remain unchanged in irx 8 anthers compared to wild type (Figure 2D, Supplemental Figures S5B,F,G). No difference was seen in irx8 endothecium walls using anti-pectin antibodies JIM5, JIM7, and CCRC-M38 (Supplemental Figures S5U-W) that recognize HG epitopes with various degrees of methylesterification, and little change was observed in this tissue using antibodies CCRC-M14, JIM13, and CCRC-M1, which recognize RG-I backbone, arabinogalactan protein (AGP), and fucosylated xyloglucan epitopes, respectively (Supplemental Figures S5X-Z).

TEM revealed smaller pollen size in irx8-5 (Figure 2J), which was confirmed by SEM (Figure 3A). While the wild-type pollen grains are uniform in size and shape, both mutant alleles of irx8 have smaller pollen grains when manually released from pollen sacs (Figure 3B). Occasionally we observed defective irx8 pollen grains under both SEM and TEM. However, since pollen development is easily affected by growth conditions, and pollen formation involves both sporophytic and gametophytic factors (Ariizumi and Toriyama, 2011), we wanted to test directly whether GAUT12 affects pollen fertility. We found that manually released $i r x 8$ pollen was able to pollinate both wild-type and heterozygous irx8 pistils, and wild-type pollen was able to 
pollinate the ir $x 8$ pistil, both producing viable seeds. Interestingly, however, manual fertilization of the homozygous irx 8 pistil with its own pollen was not successful. We observed that the irx8 inflorescence often dried soon after the stem stopped elongating, which may be due to the poor water conduction in this mutant. This may explain why manual fertilization of the $i r x 8$ pistil was unsuccessful.

We used quantitative PCR to determine if GAUT12 is expressed in pollen. Small amounts of GAUT12 transcript was detected in hydrated pollen grains and pollen tubes (Supplemental Figure S6). However, no expression of CESA4 or IRX9 transcript, genes known to encode secondary wall cellulose and xylan biosynthetic proteins, respectively (Taylor et al., 2003; Peña et al., 2007), was detected in either tissue. In contrast, relatively high levels of expression were observed for GAUT1 and CESA1 (Supplemental Figure S6B), genes known to be involved in primary cell wall pectin and cellulose biosynthesis, respectively (Arioli et al., 1998; Sterling et al., 2006; Atmodjo et al., 2011).

To further analyze whether GAUT12 affects pollen tube formation and viability, we manually fertilized wild-type pistils with pollen from the irx 8 heterozygote, a pollen population consisting of both irx 8 and wild-type pollen produced in equal amounts. The number of heterozygote vs. WT progeny from these crosses were 57:52 and 53:49 for irx8-2 and irx8-5 heterozygotes, respectively, fitting the 1:1 ratio expected for Mendelian segregation according to a $\chi^{2}$ test (Figure 3C). This result demonstrated that irx 8 pollen has normal fertility. From these results we conclude that the sterility of irx 8 mutants is due to indehiscent anthers and to the resulting inability of the mutant to release pollen for fertilization.

\section{THE irX8 MUTANT HAS LOW LIGNIN CONTENT IN BASAL STEMS AND REDUCED EXPRESSION OF MAJOR LIGNIN BIOSYNTHETIC GENES}

Reduction of lignin in the irx 8 endothecium cell layer led us to examine the lignin content in ir $x 8$ stems by histochemical staining. We found a reduction in total lignin compared to wild type (Figure 4), an observation recently confirmed by Petersen et al. (2012). Wild-type xylem vessels and fibers had thick secondary walls with evenly distributed lignin (Figures 4A,D). In contrast, interfascicular fiber cells of irx 8 mutants stained very weakly with phloroglucinol-HCl (Figure 4B), indicating a loss of G lignin. Staining with Mäule reagent revealed a brown staining in $i r x 8$ xylem cells and reduced red staining in the interfascicular fiber cells (Figure 4E), indicative of a possible but lesser reduction in $\mathrm{S}$ lignin deposition. The lignin content was recovered in the GAUT12-complemented irx8 (irx8+GAUT12) plants, as shown by staining using both stains (Figures 4C,F).

To determine whether the reduced lignin content was due to reduced biosynthesis of lignin monomers, we quantified the steady state levels of transcripts for 10 enzymes of the lignin biosynthetic pathway (Raes et al., 2003) in the basal half of irx8 stems (Figure 4G). Significantly reduced expression was observed for several key enzymes including trans-cinnamate 4-hydroxylase $(\mathrm{C} 4 \mathrm{H}), 4$-coumaroyl shikimate $3^{\prime}$-hydroxylase $\left(\mathrm{C}^{\prime} \mathrm{H}\right)$, caffeoyl CoA O-methyltransferase (CCoAOMT1), and caffeic acid/5hydroxyferulic acid $O$-methyltransferase (COMT1). We conclude that the generation of lignin precursors is likely down-regulated in the Arabidopsis irx 8 mutant.
To further evaluate lignin structure and lignin composition in irx8 stems, AIR was analyzed using pyrolysis molecular beam mass spectrometry (pyMBMS) (Sykes et al., 2008). There was a moderate but significant reduction $(9 \%)$ in total lignin which was complemented by the GAUT12-EGFP transgene (Figure $4 \mathbf{H}$ ). The reduction of total lignin in $i r x 8$ stems was largely due to a lower amount (18\% reduction) of guaiacyl $(G)$ subunits, whereas there was a relatively normal amount of $p$-hydroxyphenyl $(\mathrm{H})$ and syringyl (S) subunits in irx8 (Figure 4I). The reduction in $\mathrm{G}$ lignin resulted in an increased $\mathrm{S} / \mathrm{G}$ ratio (irx8-2: $0.9 \pm 0.09$; WT: $\left.0.7 \pm 0.06 ; P_{t \text {-test }}=0.0006\right)$. The irx $8+G A U T 12$ plants had an $\mathrm{S} / \mathrm{G}$ ratio of $0.6 \pm 0.04\left(P_{t \text {-test }}=0.011\right)$, slightly lower than that of the wild type.

We further characterized the lignification in irx8 and wild-type stems by sequentially extracting stem AIR with $50 \mathrm{mM}$ ammonium oxalate, $50 \mathrm{mM}$ sodium carbonate, $1 \mathrm{M}$ and $4 \mathrm{M} \mathrm{KOH}$, acidified sodium chlorite, and post-chlorite $4 \mathrm{M} \mathrm{KOH}$. We first used $2 \mathrm{D}{ }^{13} \mathrm{C}-{ }^{1} \mathrm{H}$ HSQC NMR spectroscopy to analyze the cell wall fraction extracted with acidified sodium chlorite, which delignifies the biomass. The aromatic region in the HSQC spectrum of the ir $x 8$ chlorite extract revealed a dramatic loss of $\mathrm{G}$ lignin $\mathrm{C} / \mathrm{H}-2,5,6$ signals (Figure 5B) compared to that of the wildtype chlorite extract (Figure 5A). This result is consistent with the reduction in $\mathrm{G}$ lignin identified by phloroglucinol- $\mathrm{HCl}$ staining (Figure 4B) and pyMBMS (Figure 4I). Consistent with the complementation result (Supplemental Figures S1, S2), G lignin cross peaks were present in the HSQC spectrum in the chlorite extract of irx8+GAUT12 complemented plants (Figure 5C). Only trace amounts of $\mathrm{H}$ and $\mathrm{S}$ lignin signals were identified in the chlorite extract across all three samples, indicating that either these monomers were lost during sample preparation or located in other wall extracts.

We thus used 2D ${ }^{13} \mathrm{C}-{ }^{1} \mathrm{H}$ HSQC NMR spectroscopy to examine all other wall extracts from both wild-type and irx8 stems, including ammonium oxalate-, sodium carbonate-, $1 \mathrm{M} \mathrm{KOH}-$, $4 \mathrm{M} \mathrm{KOH}-$, post-chlorite $4 \mathrm{M} \mathrm{KOH}(\mathrm{PC} 4 \mathrm{MKOH})$ fractions, and residual pellets. Surprisingly, we found that both the $1 \mathrm{M}$ and $4 \mathrm{M} \mathrm{KOH}$ extracts of wild type and $\operatorname{irx} 8$ contained most of the $\mathrm{H}$ lignin, with the major $\mathrm{H}$ lignin signals located in the $1 \mathrm{M}$ $\mathrm{KOH}$ extract (Figures 6A-D). The G lignin in the ir 88 mutant, although significantly reduced in amount, was found almost exclusively in the $1 \mathrm{M} \mathrm{KOH}$ extract (Figure 6B), while the G lignin in the wild type was located in both the chlorite- (Figure 5A) and $\mathrm{PC} 4 \mathrm{MKOH}$ extracts (Figure 6E). These results reveal that the $\mathrm{G}$ lignin in the $\operatorname{irx} 8$ mutant is more easily extracted than in the wild type. Only trace amounts of S lignin were found across all five wall extracts, indicating that $S$ lignin was either composed of small molecules or degraded to small molecules during sequential extractions, and thus lost during sample dialysis. The aromatic regions of wild-type and irx 8 pectin-enriched fractions, i.e., ammonium oxalate- and sodium carbonate-extracts, showed comparable amounts of $\mathrm{H}$ lignin. The cellulose-enriched pellets did not show recognizable signals for major lignin structures (Supplemental Figure S7). Compared to wild type, the lignin aliphatic (side-chain) regions in the HSQC NMR spectra of the irx 8 chlorite extract suggested a reduction in the signals for $\beta-O$ $4, \beta-5$, and $\beta-\beta$ linked lignin (Supplemental Figure S8B), which were calculated by density functional theory to be the major 

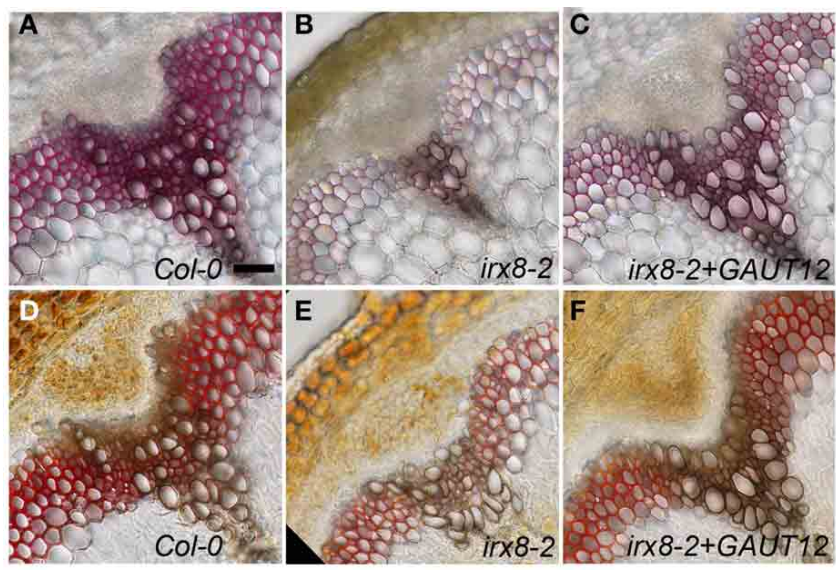

H
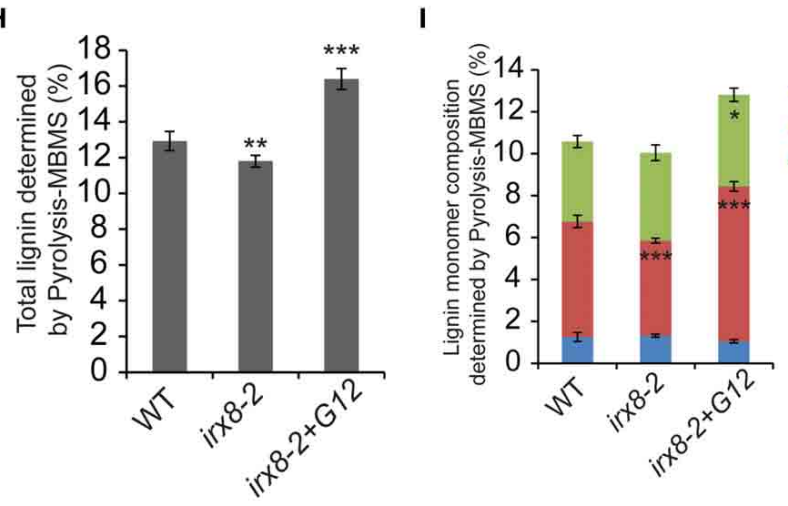

$\mathbf{G}$

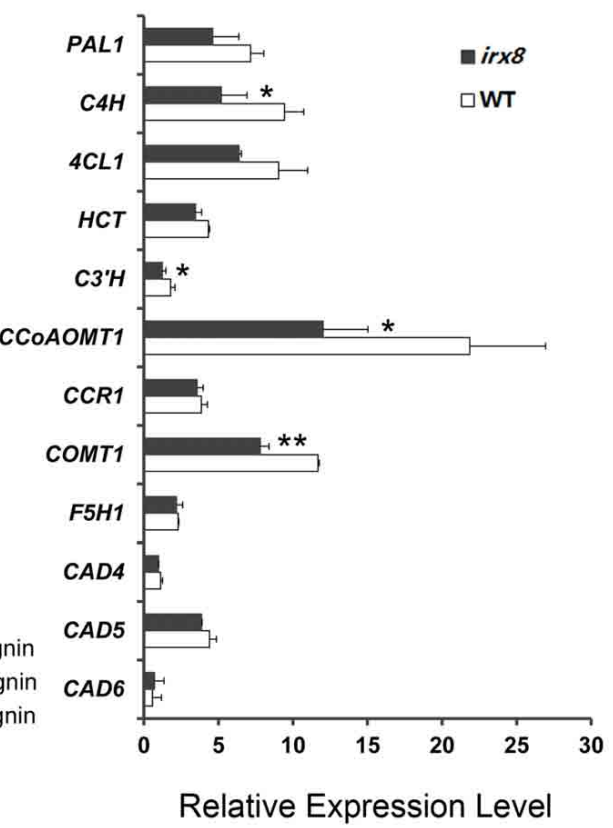

FIGURE 4 | Measurement of lignin and lignin biosynthetic gene expression in the irx8 mutant. Phloroglucinol- $\mathrm{HCl}$ staining of free-hand basal stem cross-sections of WT (A), irx8-2 (B), and irx8-2+GAUT12 (C). Mäule staining of free-hand basal stem cross-sections of WT (D), irx8-2 (E), and irx8-2+GAUT12 (F). Bar $=50 \mu \mathrm{m}$ for A-F. (G) Expression analyses in Arabidopsis WT and irx8 lower stems of lignin biosynthetic genes using Real-Time PCR. Genes labeled with asterisks have significantly lower expression in irx8-5 compared to WT $\left({ }^{*} p<0.05\right.$; $\left.{ }^{* *} p<0.01\right)$. The relative expression level of each gene was normalized using Actin2 as the reference gene and the expression of $C 3^{\prime} H$ in wild-type basal stem was set to 1 . Values are mean \pm standard deviation $(n=3)$. Lower-stem refers to the lower half of the inflorescence. $\mathrm{PAL}$, phenylalanine ammonia lyase; $\mathrm{C} 4 \mathrm{H}$, trans-cinnamate 4-hydroxylase; 4CL, 4-coumarate: CoA ligase; HCT, hydroxycinnamoyl-CoA: shikimate/quinate hydroxycinnamoyltransferase; $\mathrm{C}^{\prime} \mathrm{H}$, 4-coumaroyl shikimate 3'-hydroxylase; CCOAOMT1, caffeoyl-CoA 3-O-methyltransferase; CCR, cinnamoyl-CoA reductase; F5H, ferulate 5-hydroxylase; COMT, caffeic acid/5-hydroxyferulic acid $O$-methyltransferase; CAD, cinnamyl alcohol dehydrogenase; PER/LAC, peroxidases/laccases. (H) Total lignin (\%) determined by pyrolysis molecular beam mass spectrometry (pyMBMS) of stem alcohol insoluble residues. (I) Lignin monomer composition determined by pyMBMS. Lignin content percentage (\%) was corrected by equivalence to Klason lignin. The irx8-2 and irx8-2+GAUT12 values were compared to WT as determined by ANOVA and post-hoc t-tests with Bonferroni correction $\left({ }^{*} p<0.05 ;{ }^{* *} p<0.01 ;{ }^{* * *} p<0.001 ; n=6\right)$ for $(\mathbf{H}, \mathbf{I})$. and thermodynamically favorable linkages within lignin polymers (Sangha et al., 2012), and hence the most stable during acidic chlorite extractions.

\section{GLYCOME PROFILING INDICATES ALTERED EXTRACTABILITY OF XYLAN, PECTIN, AND AG EPITOPES IN WALLS OF THE irx8 MUTANT}

To examine cell wall glycan epitope changes associated with the loss of GAUT12 function and to correlate irx 8 wall polysaccharide epitope changes with the lignin signal alterations observed by NMR, we performed glycome profiling analyses on wall extracts from wild type, irx8, and GAUT12-complemented irx 8 (irx8+GAUT12) stems. The analyses correlated ELISA signals of different monoclonal antibody $(\mathrm{mAb})$ groups with carbohydrates released in each fraction. Cell walls (i.e., AIR) prepared from irx8 showed major differences in their glycome profiles when compared with WT walls. These differences are highlighted by dotted blocks in Figure 7.
A pronounced difference was noted in the extractability of xylan epitopes in irx8, compared to the wild type. Ammonium oxalate-, sodium carbonate-, and $1 \mathrm{M} \mathrm{KOH}$-extracts prepared from $\operatorname{ir} x 8$ walls contained significantly less xylan epitopes recognized by the xylan 4 through 7 groups of xylan-directed mAbs. Since less or similar amounts of carbohydrate mass was isolated in these extracts from irx 8 walls compared to wild-type walls, there was significantly less xylan in the oxalate-, carbonate-, and $1 \mathrm{M}$ $\mathrm{KOH}$ extracts in irx8 (Figure 7). The results indicate a loss of easily extractable xylan that is potentially associated with pectin in irx8, since these wall extracts contain large amounts of pectin. Both the $4 \mathrm{M} \mathrm{KOH}$ - and PC4MKOH extracts of irx8, however, displayed a marginal increase in binding of xylan mAbs compared to the corresponding WT extracts. The result that more xylan in the ir $x 8$ mutant was extracted under harsher conditions (i.e., with $4 \mathrm{M} \mathrm{KOH}$ and post-chlorite $4 \mathrm{M} \mathrm{KOH}$ ) correlates with the previous finding that the $4 \mathrm{M} \mathrm{KOH}$ fractions from irx 8 stems contain 


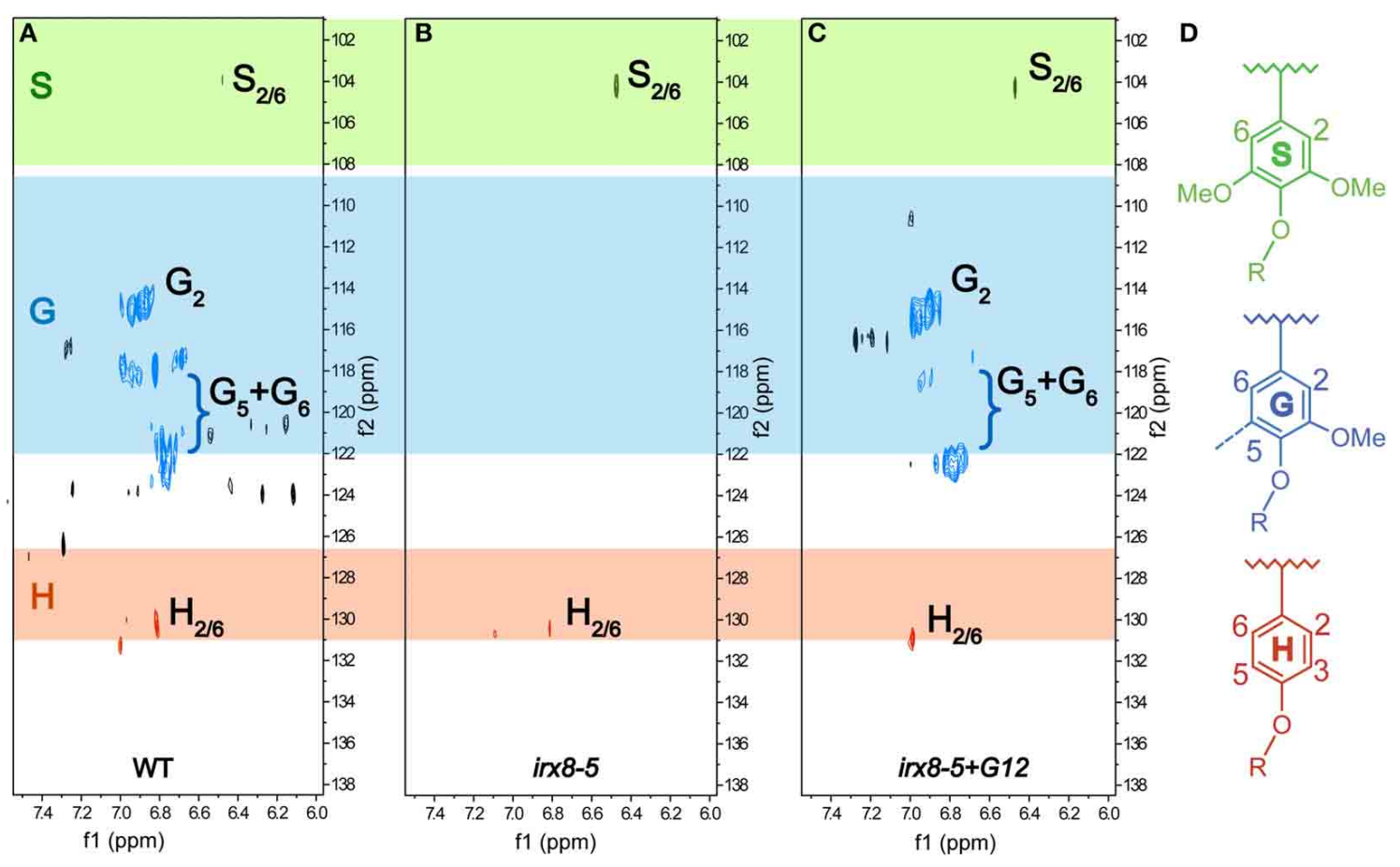

FIGURE 5 | Aromatic regions of $2 \mathrm{D}^{13} \mathrm{C}-{ }^{1} \mathrm{H}$ Heteronuclear Single-Quantum Correlation (HSOC) NMR spectra. Chlorite extracts prepared from WT (A), irx8-5 (B), and irx8-5+GAUT12 (C) stem alcohol insoluble residues (AIR) are shown. The ${ }^{13} \mathrm{C} /{ }^{1} \mathrm{H}$ signals for $\mathrm{G}$ lignin were absent in the $\mathrm{HSQC}$ spectrum of irx8-5 (B) compared to wild type (A), while the $\mathrm{G}$ lignin signals were recovered in the spectrum of irx8-5+GAUT12 (C). (D) Lignin monomer structures. some xylan of higher molecular weight than the WT counterparts (Brown et al., 2007; Peña et al., 2007).

Other differences in the glycome profile of the irx8 mutant include a reduced presence of HG backbone epitopes (those recognized by HG Backbone-I group of mAbs, Figure 7) and of arabinogalactan (AG) epitopes in the oxalate and carbonate extracts (those recognized by AG-3 and AG-4 groups of mAbs, Figure 7), as well as a reduction in pectic arabinogalactan epitopes (recognized by RG-I/AG antibodies) and AG (those recognized by the AG-4 group of mAbs) in the $1 \mathrm{M} \mathrm{KOH}$ extract (Figure 7). These results indicate a concomitant loss of pectin, AG, and xylan in these wall extracts. The complemented line irx8+GAUT12 shows a partial reversion of the glycome profile pattern to that of the WT, including an enhanced extractability of xylan, HG, and AG epitopes in the oxalate and carbonate extracts. The chlorite extracts of irx8 showed significantly enhanced levels of hemicellulose epitopes including xylan and xyloglucan (Non-Fuc XG-1 to $\mathrm{XG}-6$, Fuc-XG, and xylan-1/XG) compared to those of wild type and irx8+GAUT12. There was also a marginal increase in binding of antibodies against pectic arabinogalactan (RG-I/AG and AG2) in the irx 8 chlorite extract. This wall extract contained more mass in the irx 8 extract compared to the wild type, suggesting an increased weight ratio of carbohydrates to lignin in this extract (possibly caused by the reduction of lignin content described above; Figure 5B). An increase in pectic arabinogalac$\tan$ (RG-I/AG and AG2) epitope content was also observed in the irx8 PC4MKOH extract, which contained less mass compared to the wild type and irx8+GAUT12. These results indicate a shift in the extractability of RG-I/AG epitopes in the irx 8 mutant that may compensate for the slight reduction of these epitopes in the oxalate- and carbonate fractions. Together, these results show that irx 8 has significant changes in the extractability of glycan epitopes, particularly of xylan, pectin, and some AG.

\section{THE irx8 MUTANT EXHIBITS INCREASED RG-I LABELING AND ALTERED XYLAN LOCALIZATION PATTERNS IN FIBER CELL WALLS}

In addition to the significant reduction in xylan in irx8 fiber cell walls based on reduced immunolabeling by anti-xylan antibodies LM10 and LM11 (Figure 8; Supplemental Figures S3F, S8), there was also an unexpected increase in immunolabeling by the antibody CCRC-M14, which recognizes an RG-I backbone epitope (Figure 8). CCRC-M14 binds to an empty triangleshaped region in tricellular junctions of WT fiber cells (Figure 8). However, in 6-7-week-old irx8 basal stems, this antibody also labels the inner layer of fiber cell walls in a dotted and lamellate pattern, suggesting possible increases in the amount or accessibility of RG-I-associated cell wall epitopes in irx8 fiber cells. Consistent with this result, a slight increase in CCRC-M14 labeling was also observed in the glycome profiles in the $4 \mathrm{M} \mathrm{KOH}-$, chlorite-, and PC4MKOH extracts of irx 8 compared to the WT and irx $8+G A U T 12$ counterparts (Figure 7, green arrows). It is worth mentioning that we have also observed the loss of the CCRC-M14 labeling pattern in fiber cells in 8-week-old (or older) WT and irx8 basal stem sections, indicating that this phenotype may relate to a specific developmental stage. The GAUT12EGFP construct complemented the CCRC-M14 phenotype in 

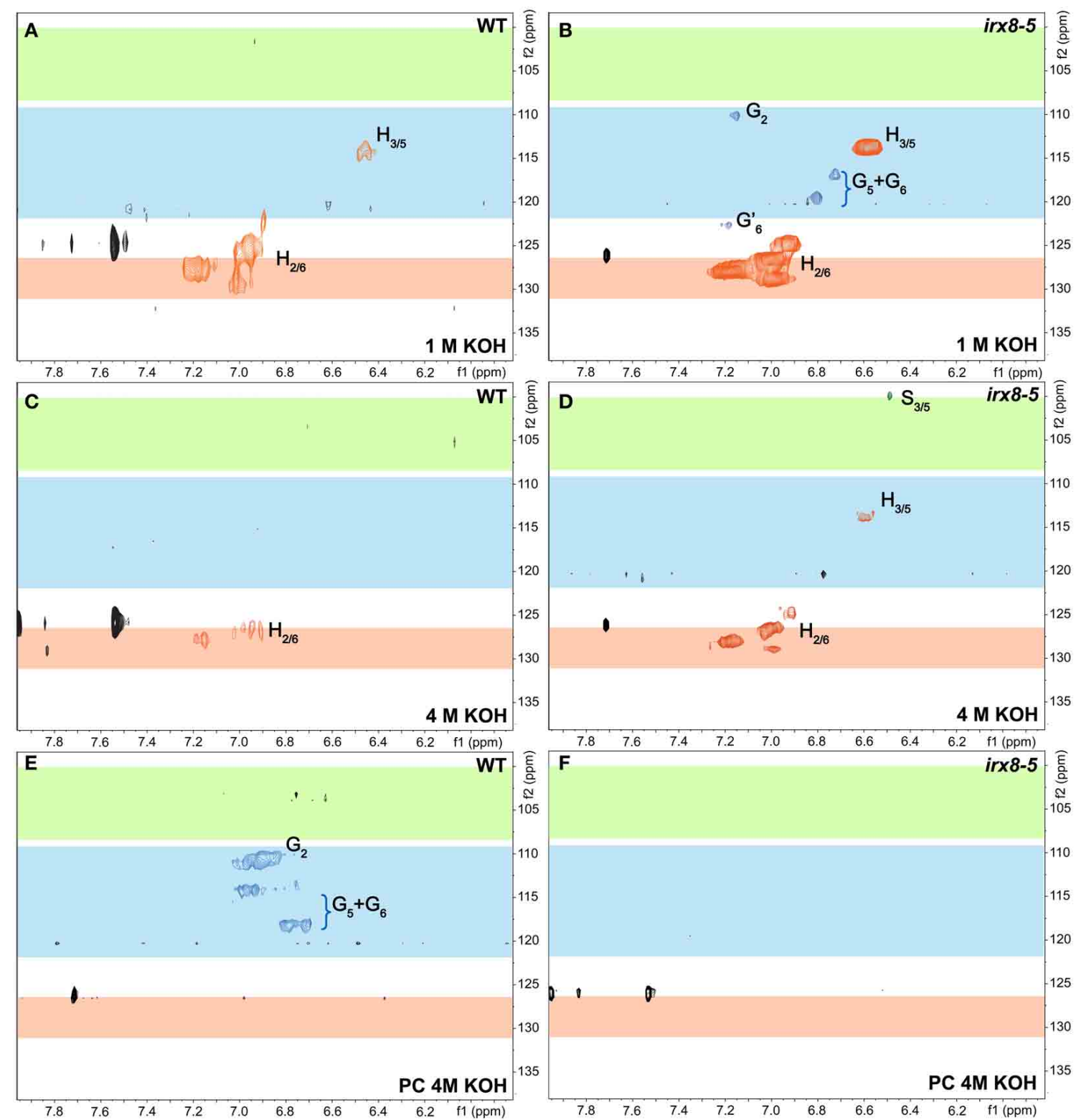

FIGURE 6 | Aromatic regions of $2 \mathrm{D}^{13} \mathrm{C}-{ }^{1} \mathrm{H}$ Heteronuclear

Single-Quantum Correlation (HSOC) NMR spectra of $1 \mathrm{M} \mathrm{KOH,} 4 \mathrm{M} \mathrm{KOH,}$ and post-chlorite-4 M KOH extracts of WT and irx8-5 mutant walls. WT

(A) and irx8-5 (B) $1 \mathrm{M} \mathrm{KOH}$ extracts. WT (C) and irx8-5 (D) $4 \mathrm{M} \mathrm{KOH}$ extracts. WT (E) and irx8-5 (F) post-chlorite $4 \mathrm{M} \mathrm{KOH}$ extracts. The signals of $\mathrm{H}$, S, and $\mathrm{G}$ lignin monomers are as labeled.

irx8 fiber cells (Figure 8). Over-expression of GAUT12 in WT (WT+GAUT12) plants, on the other hand, led to an occasional accumulation of CCRC-M14 reactive material in areas of the wall outside the triangular cell corner regions observed in the WT (Figure 8). There was, however, no obvious growth phenotype or altered sugar composition in WT vs. GAUT12 over-expression lines (Supplemental Figures S1G, S3D,H, S4, S10).

We applied a selection of six xylan-directed antibodies to basal stem sections of wild type, irx8, and GAUT12-transgenic lines (Supplemental Figure S9). Compared to WT fiber cells, there is a reduction in the labeling intensity in irx8 fibers with LM10, CCRC-M137, and CCRC-M160. LM10 binds to low-substituted xylan (McCartney et al., 2005), while both CCRC-M138 and CCRC-M160 bind to unsubstituted xylopentaose in ELISAs. The fiber walls of the irx8+GAUT12 plant are much thicker than those of irx8, but are still slightly thinner than WT fiber walls and show lower labeling intensities with LM10, LM11, and CCRC-M137, a pattern resembling those of the irx 8 mutant labeled using these three antibodies (Supplemental Figure S9). Interestingly, we noticed a double-ring labeling pattern in WT fiber cell walls labeled with CCRC-M138, a monoclonal antibody that recognizes unsubstituted xylopentaose (Figure 8). In WT fiber cells, both an inner, plasma membrane-proximal wall domain and an outer, middle lamella-proximal wall domain 


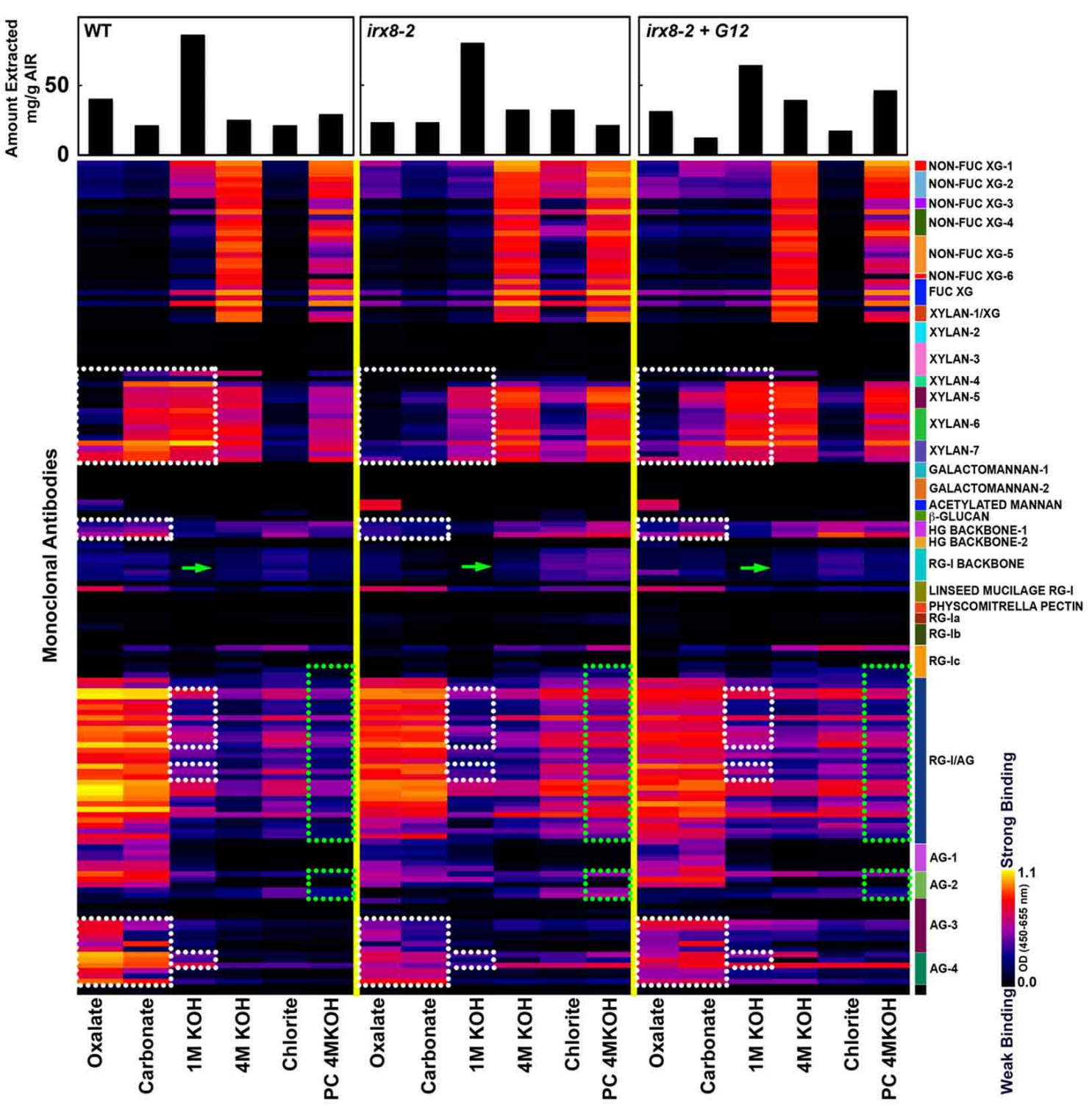

FIGURE 7 | Glycome profiling of cell walls prepared from 7/8-week-old stem tissues of irx8-2, irx8-2+GAUT12, and wild-type (WT) plants. Sequential cell wall extracts were made from respective cell wall preparations using ammonium oxalate (oxalate), sodium carbonate, potassium hydroxide ( $1 \mathrm{M}$ and $4 \mathrm{M} \mathrm{KOH})$, acidified chlorite and potassium hydroxide post chlorite $(\mathrm{PC} 4 \mathrm{MKOH})$. The resulting extracts were screened by ELISA using a suite of 155 monoclonal antibodies (mAbs) directed against diverse epitopes present on major plant cell wall glycans (See Supplemental Table S2). The ELISA binding responses are represented as heatmaps with white-red-black scale indicating the signal strength of ELISA (white, red, and dark-blue colors depict strong, medium, and no binding, respectively). The mAbs are grouped based on their specificity for cell wall glycans as shown in the panel at right hand side of the figure. The actual amount of material extracted with each extraction reagent is depicted in bar graphs at the top of the heatmaps. Glycan epitopes with reduced signals in irx 8 are outlined with white dotted blocks, while those with increased signals in irx 8 are outlined with green dotted blocks. Green arrows point to CCRC-M14 labeling. are solidly and continuously labeled with CCRC-M138, whereas xylan between these two rings, the middle layer, is mostly not labeled with this antibody. In irx8 fibers the CCRC-M138labeled double-ring, while still present as can be observed readily in cell corners, has collapsed and displays a discontinuous (dotted) pattern, which is clearly different from that of WT fiber walls. The reduced thickness or loss of the middle layer in the irx 8 mutant results in much thinner fiber cell walls. Overall, the GAUT12 construct complemented the CCRC-M138 phenotype in irx8 fiber cells (Figure 8), although the labeling intensity in the labeled wall domains appears slightly uneven (Supplemental Figure S9). CCRC-M160 shows a very similar labeling pattern to CCRC-M138 in the GAUT12-complemented (irx8+GAUT12) line, albeit its double-ring pattern is less manifest in WT and WT+GAUT12 fibers (Supplemental Figure S9). The xylan deposition changes were also reflected in the altered xylan extraction patterns identified in irx 8 by glycome profiling (Figure 7). 


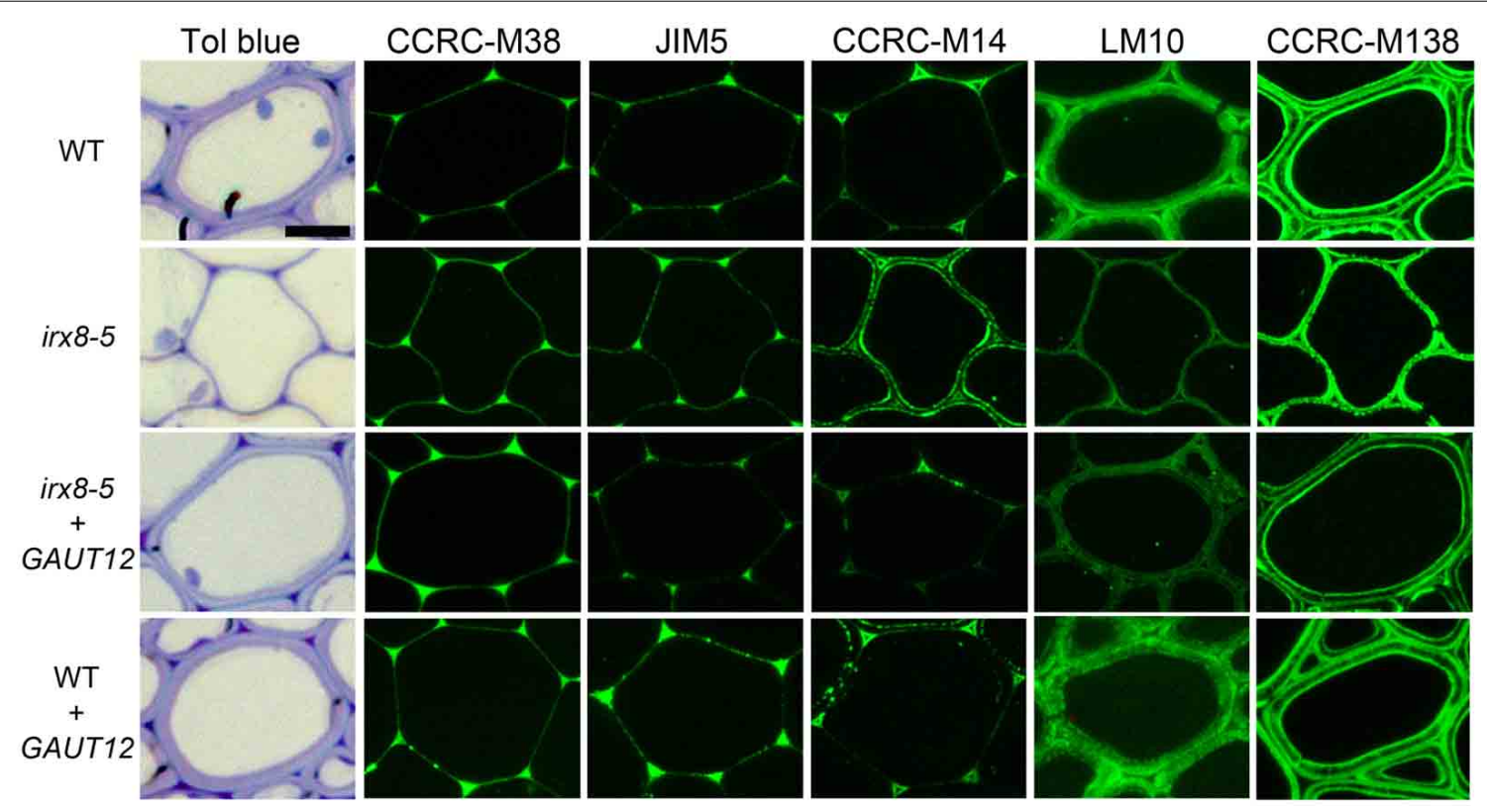

FIGURE 8 | Immunolabeling of interfascicular fiber cells from basal stems of 6-week-old wild-type (WT), irx8-5, irx8-5+GAUT12, and WT+GAUT12 plants with anti-pectin and anti-xylan antibodies. CCRC-M38 recognizes un-esterified HG, JIM5 recognizes low-esterified HG, CCRC-M14 recognizes RG-I backbone, LM10 (Xylan-6) binds to low-substituted xylan, and CCRC-M138 (Xylan-6) binds to unsubstituted xylopentaose (Pattathil et al., 2010) Bar $=10 \mu \mathrm{m}$

\section{IMMUNOABSORBED GAUT12 IS NOT AN HG:GALAT WITH CHARACTERISTICS COMPARABLE TO GAUT1}

The increased CCRC-M14 labeling in irx8 stems which suggested a change in RG-I, along with the previously reported reduction in a subfraction of HG (Persson et al., 2007), is consistent with the hypothesis that GAUT12 functions as an HG:GalAT required for secondary wall and xylan formation. To test this possibility, we measured HG:GalAT activity in microsomes from irx 8 stems which were shown to contain no GAUT12 protein (Figure 9B). The ir 8 microsomes contained $55 \%$ of the GAUT1-like HG:GalAT activity present in wildtype microsomes (Figure 9A). However, it remained unclear whether this reduction was directly due to the loss of GAUT12 activity or due to indirect effects on other enzymes, such as the HG:GalAT GAUT1, which is known to be expressed in Arabidopsis stems (Atmodjo et al., 2011). To directly test if GAUT12 has HG:GalAT activity, we generated a polyclonal anti-GAUT12 antibody that specifically recognizes GAUT12 and does not cross-react with GAUT1 or GAUT7 (Figures 9D,E; Supplemental Figure S11). LC-MS/MS was used to verify the specificity of the anti-GAUT12 antibody because the GAUT protein family contains 15 members with high sequence identity and similarity (Sterling et al., 2006). LC-MS/MS showed that peptides recovered from the protein immunoprecipitated by anti-GAUT12 are GAUT12-specific sequences and not those belonging to other GAUT proteins (Figure 9E). The antigen-purified anti-GAUT12 antibody was used to immunoabsorb-GAUT12 from detergentpermeabilized microsomes from wild-type stems (Figure 9D), and the immunoabsorbed GAUT12 was assayed for HG:GalAT activity. Neither the immunoabsorbed-GAUT12 from wild-type
(WT) stem microsomes nor the immunoabsorbed-GAUT12EGFP from Arabidopsis suspension culture cells over-expressing the GAUT12-EGFP fusion protein showed significant HG:GalAT activity (Figure 9C), although both proteins were confirmed to be present in these fractions by western blotting (Figure 9D). Thus, GAUT12 either does not have HG:GalAT activity, or its HG:GalAT activity is biochemically distinct from that of GAUT1 and cannot be assayed under the same reaction conditions.

\section{DISCUSSION}

\section{GAUT12 IS REOUIRED FOR ANTHER DEHISCENCE}

Persson et al. (2007) have described the irx 8 mutants as semisterile (irx8-1 and irx8-2) having shorter anther filaments, less pollen than the wild type, and no seeds (Persson et al., 2007). Indeed, in our hands we were not able to recover any seeds from either irx8-2 or irx8-5 plants. Other dwarf mutants with collapsed xylem phenotypes, particularly irx9 and parvus-3, have dehiscent anthers that release pollen (Supplemental Figure S4) and produce seeds under the same growth conditions. It is believed that IRX 9 is involved in xylan backbone elongation and GAUT12 together with PARVUS/GATL1 are involved in XRES biosynthesis (Brown et al., 2007; Lee et al., 2007a,b; Peña et al., 2007). Our results suggest that the function of GAUT12 may, at least in the endothecium cell layer that is critical for anther dehiscence, be distinct from that of IRX9 and PARVUS/GATL1 in regards to xylan and lignin synthesis and deposition. Alternatively, anther dehiscence in both the irx 9 and parvus-3 mutants may be due to expression of functionally redundant genes in the endothecium cell layer.

The reduction of lignin in irx 8 anther cell walls (Figures 1E,I), together with the reduction in xylan as recognized by LM10 and 


\section{A

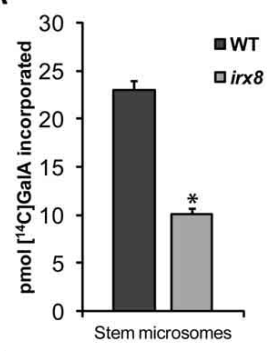

C

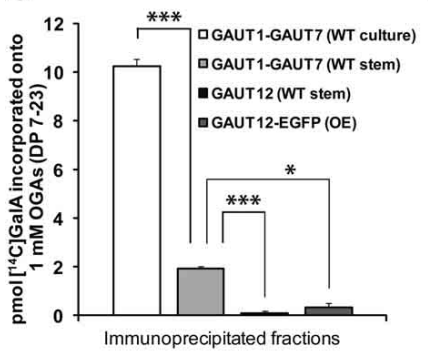

E

TM domain

51-100 EVEVISTVDT IDGDPSPCSS LACLGKRLKP KLIGRRVDSG NVPEAMYQVI 101-150 EQPLSEQELK GRSDIPQTLQ DFMSEVKRSK SDARE FAQKL KEMVTLMEQR 151-200 TRTAKIQEYL YRHVASSSIP KQLHCLALKL ANEHSINAAA $\overline{\text { RLQLPEAELV }}$ 201-250 PMLVDNNYFH FVLASDNILA ĀSVVAKSLVQ NALRPHKIVL HIITDRKTYF 251-300 PMQAWFSLHP LSPAIIEVKA LHHEDWLSKG KVPVLEAMEK DQRVRSQFRG 301-350 GSSVIVANNK ENPYVIAAKT OAISPKYNST MNHIRIHLPE IFPSINKVVE 351-400 401-450 PTIAKNENPE ECAWAYGMNV FDLAAWRRTN ISSTYYHWLD ENLKSDLSLW 451-500 QLGTLPPGLI AFHGHVQTID PFWHMLGLGY QETTSYADAE SAAVVHFNGR 501-535 AKPWLDIAFP HLRPLWAKYL DSSDRFIKSC HIRAS

\section{FIGURE 9 | Enzymatic activity assays for GAUT12 and the}

anti-GAUT12 antibody. (A) HG:GalAT activity of total microsomes $(50 \mu \mathrm{g})$ from WT and ir $x 8-5$ stems. Value $=$ mean \pm standard deviation $(n=3)$,

*indicates significant reduction of HG:GalAT activity in irx8 stem microsomes at $P_{(t-\text { test })}<0.05$. (B) Western blot analysis of immunoprecipitated (IP) GAUT12 fractions from wild-type (WT) and ir $88-5$ 7-week-old stem microsomes (500 $\mu$ g total protein) using $30 \mu \mathrm{l}$ anti-GAUT12 antibody-conjugated magnetic beads. GAUT12 protein band as indicated $(\sim 58-60 \mathrm{kDa})$ is present in WT but absent in irx8-5 microsomes. (C) HG:GalAT activity of immunoabsorbed-GAUT12 from WT stem microsomes and from GAUT12-EGFP fusion protein expressed in Arabidopsis suspension culture cells (OE). Immunoabsorbed-GAUT1:GAUT7 complex from WT suspension cell cultures and from WT stem microsomes were used as positive controls. Equal amounts of the corresponding antibody-conjugated beads $(30 \mu \mathrm{l})$ were incubated with equal amounts of TX-100 permeabilized microsomes from different tissues (500 $\mu \mathrm{g}$ total protein). Value $=$ mean \pm standard deviation $(n=3), *, * * *$ indicates significant reduction of HG:GalAT activity determined by One-Way ANOVA and post-hoc Bonferroni corrected t-test at $p<0.05$ and $p<0.001$, respectively. Immunoabsorbed-GAUT12 and GAUT12-EGFP activity are similar to background with $P_{(t-\text { test })}$ values $=0.44$ and 0.22 , respectively. All enzyme activity assays were repeated at least twice. One set of representative results is shown. (D) Western blots showing the presence of GAUT12, GAUT1, and GAUT7 in the corresponding immunoabsorbed fractions from WT stem tissues. GAUT12 was immunoabsorbed by the anti-GAUT12 antibody, and the GAUT1:GAUT7 complex was

immunoabsorbed by the anti-GAUT7 antibody. Arabidopsis culture expressed-GAUT12-EGFP ( $86 \mathrm{KDa}$ ) fusion protein in the anti-GAUT12 immunoabsorbed fractions used in reactions in (C) shown in the lower panel. (E) GAUT12 protein sequence (535-a.a.). The antigenic-peptide used to generate the anti-GAUT12 antibody is highlighted in yellow. Peptides identified in GAUT12-IP fractions by LC-MS/MS are labeled in blue with underlines. Boxed sequence indicates the GAUT12 transmembrane (TM) domain predicted by TmHMM_v2.
LM11 in endothecium cell walls (Figures 2F,G), contributes to a lack of secondary wall thickening in the irx8 endothecium layer (Figure 2J). Consequently, relatively low tension during anther wall dehydration leads to indehiscent anthers in irx 8 mutants. The pollen grains produced by ir $x 8$, albeit smaller in size (Figure 3B), are viable upon manual release and able to fertilize both wild-type and ir $x 8$ heterozygote pistils. Furthermore, manual fertilization of wild-type pistils with ir $x 8$ heterozygote pollen demonstrated that irx8 pollen has similar viability to wild-type pollen (Figure 3C). Thus, the function of GAUT12 is not essential for pollen viability and fertilization. Prior published transcriptomic and proteomic analyses of pollen and pollen tubes have not detected GAUT12 transcript or protein in these tissues (Wang et al., 2008; Zou et al., 2009). However, using quantitative RT-PCR we detected low GAUT12 expression in hydrated pollen grains and elongating pollen tubes (Supplemental Figure S6), suggesting a potential role of GAUT12 in pollen, perhaps associated with pollen size (Figure 3B).

\section{LACK OF GAUT12 FUNCTION RESULTS IN REDUCED AMOUNTS AND ALTERED EXTRACTABILITY OF G LIGNIN IN ARABIDOPSIS STEM}

We identified a reduction of lignin in the irx 8 mutant and used immunohistochemical staining (Figures 4B,E), pyMBMS (Figures 4H,I), and 2D ${ }^{13} \mathrm{C}-{ }^{1} \mathrm{H}$ HSQC NMR spectroscopy (Figure 5) to characterize this mutant phenotype in detail as well as study the possible connections between lignin and xylan deposition. In the semi-quantitative $2 \mathrm{D}{ }^{13} \mathrm{C}-{ }^{1} \mathrm{H}$ HSQC NMR analyses, only trace amounts of $\mathrm{H}$ and $\mathrm{S}$ lignin were found in chlorite extracts from the WT, irx8-5, and ir $x 8-5+G A U T 12$ (Figure 5). $\mathrm{H}$ lignin signals, however, were identified in the oxalate-, carbonate-, $1 \mathrm{M}$ and $4 \mathrm{M} \mathrm{KOH}$ extracts of both WT and irx8, with the major signals located in the $1 \mathrm{M} \mathrm{KOH}$ extracts (Figure 6; Supplemental Figure S7). These results suggest that $\mathrm{H}$ lignin is present in the pectin and hemicellulose-enriched wall fractions. The presence of $\mathrm{H}$ lignin in pectin fractions is consistent with the observation that $\mathrm{H}$ lignin is deposited in middle lamella and cell wall corners (Donaldson, 2001) and hence co-extracted with pectin. The observation that a major portion of the $\mathrm{H}$ lignin signals were found in the $1 \mathrm{M} \mathrm{KOH}$ fraction suggests either that this portion of $\mathrm{H}$ lignin is directly or indirectly (e.g., via pectin) connected to the $\mathrm{KOH}$-solubilized xylan or xyloglucan in these fractions, or that $\mathrm{H}$ lignin is connected through alkaline-labile ester linkages (Balakshin et al., 2011), and thus, extracted in alkaline buffers. Specific linkages between the hemicellulose/pectin/H lignin, however, remain to be determined. $\mathrm{H}$ lignin signals in $i r x 8$ appeared to be slightly more prominent than in the WT, possibly due to the lower amount of xylan in the $1 \mathrm{M} \mathrm{KOH}$ extract of irx8 (Figure 7), resulting in an increased lignin to xylan weight ratio. The total amount of $\mathrm{H}$ lignin in irx8, however, was similar to that of WT as determined by pyMBMS (Figure 4I). Thus, the results do not support a role for GAUT12 in producing a structure required for $\mathrm{H}$ lignin deposition.

It is estimated that the chlorite extractions conducted in this study could remove $50-80 \%$ of total lignin based on studies of the efficiency of acidic sodium chlorite treatment in the removal of lignin in black spruce, switchgrass, and poplar (Ahlgren and Goring, 1971; Kumar et al., 2013). Therefore, the lignin signals 
observed in the chlorite- and PC4MKOH extracts by HSQC NMR were likely portions of the residual lignin (20-50\%) recovered from each extraction at, or after, the sodium chlorite treatment. This may explain why we did not identify $\mathrm{S}$ lignin signals across all fractions, although it is unclear whether $S$ lignin is composed of smaller molecules and depleted during sequential extractions and dialyses. The G lignin in WT was identified mostly in the chlorite- and $\mathrm{PC} 4 \mathrm{MKOH}$ extracts (Figures 5A, 6E), indicating that a portion of $\mathrm{G}$ lignin is linked to wall polysaccharides via alkaline-resistant linkages, such as benzyl ethers and phenyl glucosides, and that only harsh conditions like acidic sodium chlorite are able to degrade these linkages. In irx8, however, the chlorite fraction is nearly depleted of G lignin signals (Figure 5B). Surprisingly, the bulk of the $\operatorname{irx} 8 \mathrm{G}$ lignin signals, albeit much reduced in amount compared to the WT, was found in the $1 \mathrm{M}$ $\mathrm{KOH}$ extract (Figure 6B), a wall fraction released prior to the acidic chlorite treatment, clearly indicating an altered $G$ lignin extractability in the irx8 mutant. It remains unknown, however, whether there is a portion of G lignin in the irx 8 mutant that does not withstand the acidic chlorite treatment and hence is removed during subsequent dialysis. Overall the results are consistent with the reduced phloroglucinol- $\mathrm{HCl}$ staining of $\operatorname{ir} x 8$ stem cross-sections (Figure 4B) and the reduced G lignin monomer content identified by pyMBMS (Figure 4I). Our results suggest that in the irx8 mutant either (i) the $\mathrm{G}$ lignin linkages themselves or (ii) the polymers to which the G lignin is connected are partially alkaline-labile, and hence, more easily extracted from the walls in this mutant than in the WT.

\section{INTERDEPENDENCE OF LIGNIN, PECTIN AND XYLAN IN WALL BIOGENESIS}

The pleiotropic effects of irx 8 on xylan, lignin, and pectin during secondary wall formation confirms that normal wall biogenesis is dependent on an interaction between different cell wall polymers and suggests that there may be a requisite order in which they are deposited in muro. Lignification, which creates a relatively rigid and impermeable resin within the polysaccharide network of the wall, has been proposed to occur in two distinct stages. In the primary wall it appears that lignin deposition starts as early as during the formation of the middle lamella at the cell plate, followed by deposition at the cell wall corners-a sequence that has been taken to imply the existence of possible pectin initiation/nucleation sites for primary wall lignin deposition (Donaldson, 2001). Our data demonstrate that Arabidopsis interfascicular fiber tricellular junctions are filled with un-esterified to low-esterified HG, as recognized in a solid triangle shape by antiHG antibodies CCRC-M38 and JIM5 (Figure 8). This tricellular junction triangle also contains a small amount of high-esterified HG as labeled by JIM7 (Supplemental Figure S9). RG-I backbone, as recognized by CCRC-M14, appears to be present toward the outer layer of this HG triangle, where labeling with this antibody is observed as an empty triangle at the tricellular junction (Figure 8). The lignin in this tricellular junction area was solidly stained for both $\mathrm{G}$ and $\mathrm{S}$ lignin in irx8 fibers (Figures 4B,E), suggesting that this process is unlikely to have been affected by the mutation in GAUT12. In a second stage of lignification, secondary cell wall lignin is deposited in specific, terminally differentiated cell types (Donaldson, 2001). We showed by colorimetric staining that both $\mathrm{G}$ and $\mathrm{S}$ lignin are reduced in the irx 8 xylem vessels and fiber cells, apparently due to the reduction in wall thicknesses in these cells. The G lignin in irx8 is significantly reduced (Figures 4I, 5B), and released in the $1 \mathrm{M} \mathrm{KOH}$ extract (Figure 6B) rather than in the chlorite- and PC4MKOH extracts as occurs in the WT (Figures 5A, 6E), suggesting a possible correlation between the xylan reduction and lignin alteration in irx8. We also found a significant reduction in expression of four key lignin biosynthetic enzymes $\left(\mathrm{C} 4 \mathrm{H}, \mathrm{C}^{\prime} \mathrm{H}, \mathrm{CCoAOMT} 1\right.$, and COMT1) that may lead to reduced lignin precursor production in $i r x 8$ (Figure 4G). These results are consistent with a reduction in total lignin in irx8 walls, in particular through a reduction in G lignin. Our data support the proposition that lignin formation is downregulated in a xylan defective mutant, and that reduced GAUT12 function affects both xylan and lignin deposition.

It has been reported that loss of the primary cellulose synthase subunit CESA 3 in the eli1 mutant is associated with ectopic lignin deposition and increased defense responses (Cano-Delgado et al., 2003), which was interpreted as an effort by the cells to maintain cell wall integrity. By contrast, a decrease in lignin was observed in the $\operatorname{ir} x 8$ mutant, and reportedly also in the $\operatorname{ir} x 7$ and irx9 mutants (Petersen et al., 2012). These observations show that loss of matrix polysaccharides causes a reduction in lignin in such irx mutants and suggest that matrix polysaccharides provide a different structural function than cellulose during lignification. The reduction of lignin in the ir 8 mutant may be a secondary effect due to the $\sim 60 \%$ loss of xylan in irx8, since linkages between xylan and lignin have been reported. For example, 4-Omethylglucuronoxylan is the major carbohydrate linked to lignin in wood (Yuan et al., 2011) and xylan and lignin are linked via ferulate esters in maize (Grabber et al., 2000). The exact lignincarbohydrate linkages in the irx 8 mutant are currently under investigation.

\section{GAUT12 FUNCTION IS REOUIRED TO ESTABLISH A LAMELLATE STRUCTURE IN THE SECONDARY CELL WALL}

We observed interesting lamellate-like patterns of xylan labeling in wild-type fiber cell walls upon immunolabeling with selected xylan-directed antibodies (Figure 8; Supplemental Figure S9). These results suggest that the deposition of different xylan structure may be spatially controlled. LM10 and LM11 bind to xylooligomers as small as a disaccharide, while CCRC-M149 binding requires an unsubstituted xylotriose structure, and CCRC-M138 and CCRC-M160 require unsubstituted xylopentaose for recognition (S. Pattathil, U. Avci, and M.G. Hahn, unpublished results). In wild-type fiber cells, LM10 labeling is more intense on the side of the fiber wall adjacent to adjoining cells, and the labeling decreases in intensity toward the lumen side of the wall. LM11 has an overall dotted labeling pattern that covers the entire secondary wall in fiber cells. CCRC-M137 and CCRC-M149 show comparable and relatively even labeling throughout fiber walls. All four antibodies had reduced labeling in irx8 fiber cell walls due either to a reduced number of epitopes recognized by these antibodies or to reduced thickness of the wall. The fiber cells in GAUT12complemented (irx8+GAUT12) plants, however, show a partial restoration of the WT labeling patterns with LM10, LM11, and 
CCRC-M137. Although irx8+GAUT12 fibers have thicker walls than those of irx8, the lower labeling intensity (i.e., epitope density) with LM10, LM11, and CCRC-M137 in irx8+GAUT12 fibers resembles that of the irx 8 mutant (Supplemental Figure S9). In contrast, CCRC-M149 labels a thinner wall in irx8 fibers with similar intensity (i.e., epitope density) in both $\operatorname{irx} 8$ and irx8+GAUT12 plants compared to WT plants, suggesting that the CCRC-M149-reactive xylan was perhaps indirectly affected by the irx8 mutation. In other words, the reduction in CCRC-149 labeling in irx 8 fiber walls is likely due to reduced wall thickness and not reduced epitope density.

The most interesting labeling pattern was observed using antibodies CCRC-M138 and CCRC-M160, which showed doublering labeling patterns in wild-type fiber cell walls (Figure 8; Supplemental Figure S9). The pattern suggests that the middle layer of wild-type fiber secondary walls between the labeled double rings contains xylan (as recognized by CCRC-M149) with fewer regions of unsubstituted xylan as recognized by CCRCM138. The double-ring still exists in irx8 fiber cell walls, as can be clearly seen at cell corners, but it has collapsed in ir $x 8$ fiber cell walls due to reduced thickness of the middle layer material of the wall. In addition, the texture of the CCRC-M138 labeling in irx8 fiber walls is different than in the WT, indicating a re-organized secondary wall with an altered configuration of xylan deposition in the irx 8 mutant. This was also reflected in the altered xylan extraction patterns in irx 8 observed by glycome profiling, revealing that significantly less xylan was extracted in the ammonium oxalate-, sodium carbonate-, and $1 \mathrm{M} \mathrm{KOH}$ extracts in the irx8 mutant walls compared to WT walls (Figure 7). It is unclear why CCRC-M138 labeling is slightly uneven in the GAUT12complemented line (Supplemental Figure S9). A possible explanation is that the $35 \mathrm{~S}$ promoter drives an inconsistent expression of GAUT12 leading to the uneven production of corresponding xylan epitopes.

The cell wall immunolabeling patterns in fiber cell cross sections obtained using the six monoclonal antibodies reactive against diverse xylan epitopes indicates that GAUT12 is required for the formation of a middle xylan-containing layer located between an outer (middle lamella-proximal) and an inner (plasma membrane-proximal) xylan layer. These three layers are clearly seen in cross sections of WT fiber cells immunolabeled using antibodies LM11, CCRC-M138 and CCRC-M160. Immunolabeling of fiber cell cross sections of the irx 8 mutant reveals a loss of the middle xylan-containing layer and a collapse of the inner and outer xylan layers onto each other. Since several of the xylan-directed antibodies label the middle layer, as well as the outer and inner xylan layers (e.g., CCRC-M137 and CCRCM149), it is clear that the middle layer contains xylan. Thus, the lack of the middle layer, and the retention of the outer and inner xylan layers, albeit in a discontinuous pattern in the irx 8 mutants, indicate that GAUT12 is required to produce a WT xylan architecture and that, in the absence of GAUT12, this architecture is either not made or collapses. The current results do not clarify whether the reduction in xylan in the irx 8 mutant is due to the inability of the plant to make a subfraction of xylan that requires GAUT12 for synthesis, or rather, whether the absence of GAUT12 results in an altered xylan architecture that leads to an accumulation of surplus xylan which acts as a negative signal to down-regulate xylan synthesis.

\section{GAUT12 FUNCTION}

How may the complex phenotype of irx 8 mutants be explained? The reduction in $\alpha$-1,4-linked GalA in the endopolygalacturonase/pectin methylesterase (EPG/PME)-accessible fraction of irx 8 mutant walls raises the possibility that GAUT12 is involved in the synthesis of an as-yet-to-be-determined HG species. Such a GAUT12-dependent HG may be tightly associated with xylan and, when missing, disrupt xylan biogenesis and/or deposition (Persson et al., 2007). Such GAUT12-dependent pectin and/or xylan may further be a foundation upon which $G$ lignin accumulates.

Recently, Tan et al. (2013) discovered a novel cell wall structure isolated from Arabidopsis suspension cell cultures named ARABINOXYLAN PECTIN ARABINOGALACTAN PROTEIN1 (APAP1), which contains a unique proteoglycan with xylan connected to stretches of RG-I that are flanked by short HG oligomers (Tan et al., 2013). Although we show in the present study that GAUT12 does not appear to have GAUT1-like HG:GalAT activity, i.e., GAUT12 does not catalyze the addition of GalA from UDP- $\alpha$ GalA onto oligogalacturonide (DP 7-23) acceptors (Figure 9C), a possible function for GAUT12 is the synthesis of such an HG sub-domain using the RG-I sub-domain (oligomer) as a primer for synthesis of an APAP1-like structure in fiber walls. This hypothesis is consistent with the significant decreases in Xyl and GalA in irx8 stem cell walls (Supplemental Figure S2C) and with the increased immunolabeling of fiber walls with the RGI backbone-reactive antibody CCRC-M14 (Figure 8). Although there is no obvious change in the wall glycosyl residue composition when GAUT12 is over-expressed in the WT background (Supplemental Figure S10E), GAUT12 over-expression may lead to increased production of RG-I/HG sub-domains which may overload the capacity of the xylan biosynthesis machinery, resulting in accumulation of the CCRC-M14 reactive material at the plasma membrane as observed in the WT+GAUT12 fiber cell walls (Figure 8).

Alternatively, the reduction in the irx 8 mutant of the xylan reducing end sequence [XRES, $\beta$-D-Xyl $p-(1 \rightarrow 3)-\alpha-\mathrm{L}-$ Rhap-(1 $\rightarrow 2)-\alpha-D-G a l p A-(1 \rightarrow 4)-D-X y l p]$ led to speculations that GAUT12 may catalyze the addition of GalA into the nascent XRES (Peña et al., 2007). However, it remains unknown how XRES synthesis is initiated or whether it acts as a primer or terminator during xylan biosynthesis (York and O'Neill, 2008). GAUT12 appears not to have any functional homologs in graminaceous monocots (Caffall et al., 2009; Yin et al., 2010). The absence of XRES in grass species examined to date (Kulkarni et al., 2012) would be consistent with a function of GAUT12 in the synthesis of this structure. So far, however, we were not able to demonstrate that GAUT12 adds the GalA into the XRES. Further study is required to identify GAUT12 enzymatic function, as well as its role in the carbohydrate-lignin connection.

In summary, we demonstrate that GAUT12 has a role in anther dehiscence and affects the amount of G lignin and its connectivity to xylan in Arabidopsis. Our work shows that a 
mutation in a single glycosyltransferase leads to alterations in xylan, pectin, and lignin, thereby providing further evidence for possible associations or connections between different wall polymers. Although the catalytic activity of GAUT12 remains to be determined, we have shown that GAUT12 is not an HG:GalAT with substrate specificities comparable to GAUT1 and that the product of GAUT12 may connect to a structure that contains RG-I and that is required for native xylan architecture in the secondary cell wall.

\section{ACKNOWLEDGMENTS}

We thank Maor Bar-Peled for the 35tlegfps2\#4 plasmid as the base for the GAUT12-EGFP (GAUT12) constructs. This research was supported by the Department of Energy Center Grant DOE DE-FG02-09ER20097 and BioEnergy Science Center Grant DE-AC05-00OR22725. The BioEnergy Science Center is a US Department of Energy Bioenergy Research Center supported by the Office of Biological and Environmental Research in the Department of Energy Office of Science. The generation of the CCRC series of glycan-directed monoclonal antibodies used in this work was supported by the National Science Foundation Plant Genome Program (Awards DBI-0421683 and IOS-0923992 to Michael G. Hahn).

\section{SUPPLEMENTARY MATERIAL}

The Supplementary Material for this article can be found online at: http://www.frontiersin.org/journal/10.3389/fpls.2014.00357/ abstract

\section{REFERENCES}

Ahlgren, P. A., and Goring, D. A. I. (1971). Removal of wood components during chlorite delignification of black spruce. Can. J. Chem. 49, 1272-1275. doi: 10.1139/V71-207

Albersheim, P., Nevins, D. J., English, P. D., and Karr, A. (1967). A method for the analysis of sugars in plant cell-wall polysaccharides by gas-liquid chromatography. Carbohydr. Res. 5, 340-345.

Ariizumi, T., and Toriyama, K. (2011). Genetic regulation of sporopollenin synthesis and pollen exine development. Annu. Rev. Plant Biol. 62, 437-460. doi: 10.1146/annurev-arplant-042809-112312

Arioli, T., Peng, L., Betzner, A. S., Burn, J., Wittke, W., Herth, W., et al. (1998). Molecular analysis of cellulose biosynthesis in Arabidopsis. Science 279, 717-720. doi: 10.1126/science.279.5351.717

Atmodjo, M. A., Sakuragi, Y., Zhu, X., Burrell, A. J., Mohanty, S. S., Atwood, J. A. 3rd., et al. (2011). Galacturonosyltransferase (GAUT)1 and GAUT7 are the core of a plant cell wall pectin biosynthetic homogalacturonan:galacturonosyltransferase complex. Proc. Natl. Acad. Sci. U.S.A. 108, 20225-20230. doi: 10.1073/pnas.1112816108

Avci, U., Pattathil, S., and Hahn, M. G. (2012). Immunological approaches to plant cell wall and biomass characterization: immunolocalization of glycan epitopes. Methods Mol. Biol. 908, 73-82. doi: 10.1007/978-1-61779-956-3_7

Balakshin, M., Capanema, E., Gracz, H., Chang, H. M., and Jameel, H. (2011). Quantification of lignin-carbohydrate linkages with high-resolution NMR spectroscopy. Planta 233, 1097-1110. doi: 10.1007/s00425-011-1359-2

Boerjan, W., Ralph, J., and Baucher, M. (2003). Lignin biosynthesis. Annu. Rev. Plant Biol. 54, 519-546. doi: 10.1146/annurev.arplant.54.031902. 134938

Bouton, S., Leboeuf, E., Mouille, G., Leydecker, M. T., Talbotec, J., Granier, F., et al. (2002). QUASIMODO1 encodes a putative membrane-bound glycosyltransferase required for normal pectin synthesis and cell adhesion in Arabidopsis. Plant Cell 14, 2577-2590. doi: 10.1105/tpc.004259

Bowman, J. (1994). Arabidopsis: an Atlas of Morphology and Development. London: Springer, Limited, 2011.
Brown, D. M., Goubet, F., Wong, V. W., Goodacre, R., Stephens, E., Dupree, P., et al. (2007). Comparison of five xylan synthesis mutants reveals new insight into the mechanisms of xylan synthesis. Plant J. 52, 1154-1168. doi: 10.1111/j.1365-313X.2007.03307.x

Brown, D. M., Zeef, L. A., Ellis, J., Goodacre, R., and Turner, S. R. (2005). Identification of novel genes in Arabidopsis involved in secondary cell wall formation using expression profiling and reverse genetics. Plant Cell 17, 2281-2295. doi: 10.1105/tpc.105.031542

Caffall, K. H., Pattathil, S., Phillips, S. E., Hahn, M. G., and Mohnen, D. (2009). Arabidopsis thaliana T-DNA mutants implicate GAUT genes in the biosynthesis of pectin and xylan in cell walls and seed testa. Mol. Plant 2, 1000-1014. doi: $10.1093 / \mathrm{mp} / \mathrm{ssp} 062$

Cano-Delgado, A., Penfield, S., Smith, C., Catley, M., and Bevan, M. (2003). Reduced cellulose synthesis invokes lignification and defense responses in Arabidopsis thaliana. Plant J. 34, 351-362. doi: 10.1046/j.1365-313X.2003.01729.x

Chapple, C. C., Vogt, T., Ellis, B. E., and Somerville, C. R. (1992). An Arabidopsis mutant defective in the general phenylpropanoid pathway. Plant Cell 4, 1413-1424. doi: 10.1105/tpc.4.11.1413

Clough, S. J., and Bent, A. F. (1998). Floral dip: a simplified method for Agrobacterium-mediated transformation of Arabidopsis thaliana. Plant J. 16, 735-743. doi: 10.1046/j.1365-313x.1998.00343.x

Dardelle, F., Lehner, A., Ramdani, Y., Bardor, M., Lerouge, P., Driouich, A., et al. (2010). Biochemical and immunocytological characterizations of Arabidopsis pollen tube cell wall. Plant Physiol. 153, 1563-1576. doi: 10.1104/pp.110. 158881

Demartini, J. D., Pattathil, S., Avci, U., Szekalski, K., Mazumder, K., Hahn, M. G., et al. (2011). Application of monoclonal antibodies to investigate plant cell wall deconstruction for biofuels production. Energy Environ. Sci. 4, 4332-4339. doi: 10.1039/clee02112e

Doelling, J. H., and Pikaard, C. S. (1993). Transient expression in Arabidopsis thaliana protoplasts derived from rapidly established cell-suspension cultures. Plant Cell Rep. 12, 241-244. doi: 10.1007/BF00237127

Donaldson, L. A. (2001). Lignification and lignin topochemistry-an ultrastructural view. Phytochemistry 57, 859-873. doi: 10.1016/S0031-9422(01)00049-8

Donaldson, L. A., and Knox, J. P. (2012). Localization of cell wall polysaccharides in normal and compression wood of radiata pine: relationships with lignification and microfibril orientation. Plant Physiol. 158, 642-653. doi: 10.1104/pp.111.184036

Doong, R. L., and Mohnen, D. (1998). Solubilization and characterization of a galacturonosyltransferase that synthesizes the pectic polysaccharide homogalacturonan. Plant J. 13, 363-374. doi: 10.1046/j.1365-313X.1998.00042.x

Evans, R. J., and Milne, T. A. (1987). Molecular characterization of the pyrolysis of biomass.1. fundamentals. Energy Fuels 1, 123-137. doi: 10.1021/Ef00002a001

Grabber, J. H., Ralph, J., and Hatfield, R. D. (2000). Cross-linking of maize walls by ferulate dimerization and incorporation into lignin. J. Agric. Food Chem. 48, 6106-6113. doi: 10.1021/jf0006978

Guo, D., Chen, F., Inoue, K., Blount, J. W., and Dixon, R. A. (2001). Downregulation of caffeic acid 3-O-methyltransferase and caffeoyl CoA 3$O$-methyltransferase in transgenic alfalfa. impacts on lignin structure and implications for the biosynthesis of G and S lignin. Plant Cell 13, 73-88. doi: $10.1105 /$ tpc. 13.1 .73

Jeffries, T. W. (1990). Biodegradation of lignin-carbohydrate complexes. Biodegradation 1, 163-176. doi: 10.1007/bf00058834

Jiang, N., Pu, Y. Q., Samuel, R., and Ragauskas, A. J. (2009). Perdeuterated pyridinium molten salt (ionic liquid) for direct dissolution and NMR analysis of plant cell walls. Green Chem. 11, 1762-1766. doi: 10.1039/b913609f

Jung, K. W., Oh, S. I., Kim, Y. Y., Yoo, K. S., Cui, M. H., and Shin, J. S. (2008). Arabidopsis histidine-containing phosphotransfer factor 4 (AHP4) negatively regulates secondary wall thickening of the anther endothecium during flowering. Mol. Cells 25, 294-300.

Kim, H., and Ralph, J. (2010). Solution-state 2D NMR of ball-milled plant cell wall gels in DMSO- $d_{6} /$ pyridine- $d_{5}$. Org. Biomol. Chem. 8, 576-591. doi: 10.1039/b916070a

Ko, J. H., Kim, W. C., and Han, K. H. (2009). Ectopic expression of MYB46 identifies transcriptional regulatory genes involved in secondary wall biosynthesis in Arabidopsis. Plant J. 60, 649-665. doi: 10.1111/j.1365-313X.2009.03989.x

Kong, Y., Zhou, G., Avci, U., Gu, X., Jones, C., Yin, Y., et al. (2009). Two poplar glycosyltransferase genes, PdGATL1.1 and PdGATL1.2, are functional 
orthologs to PARVUS/AtGATL1 in Arabidopsis. Mol. Plant 2, 1040-1050. doi: $10.1093 / \mathrm{mp} / \mathrm{ssp} 068$

Kulkarni, A. R., Peña, M. J., Avci, U., Mazumder, K., Urbanowicz, B. R., Pattathil, S., et al. (2012). The ability of land plants to synthesize glucuronoxylans predates the evolution of tracheophytes. Glycobiology 22, 439-451. doi: 10.1093/glycob/cwr117

Kumar, R., Hu, F., Hubbell, C. A., Ragauskas, A. J., and Wyman, C. E. (2013). Comparison of laboratory delignification methods, their selectivity, and impacts on physiochemical characteristics of cellulosic biomass. Bioresour. Technol. 130, 372-381. doi: 10.1016/j.biortech.2012.12.028

Lao, N. T., Long, D., Kiang, S., Coupland, G., Shoue, D. A., Carpita, N. C., et al. (2003). Mutation of a family 8 glycosyltransferase gene alters cell wall carbohydrate composition and causes a humidity-sensitive semisterile dwarf phenotype in Arabidopsis. Plant Mol. Biol. 53, 647-661. doi: 10.1023/B:PLAN.0000019074.60542.6c

Leboeuf, E., Guillon, F., Thoiron, S., and Lahaye, M. (2005). Biochemical and immunohistochemical analysis of pectic polysaccharides in the cell walls of Arabidopsis mutant QUASIMODO 1 suspension-cultured cells: implications for cell adhesion. J. Exp. Bot. 56, 3171-3182. doi: 10.1093/jxb/eri314

Lee, C., O’Neill, M. A., Tsumuraya, Y., Darvill, A. G., and Ye, Z. H. (2007a). The irregular xylem 9 mutant is deficient in xylan xylosyltransferase activity. Plant Cell Physiol. 48, 1624-1634. doi: 10.1093/pcp/pcm135

Lee, C., Zhong, R., Richardson, E. A., Himmelsbach, D. S., McPhail, B. T., and Ye, Z. H. (2007b). The PARVUS gene is expressed in cells undergoing secondary wall thickening and is essential for glucuronoxylan biosynthesis. Plant Cell Physiol. 48, 1659-1672. doi: 10.1093/pcp/pcm 155

Lewis, N. G., and Yamamoto, E. (1990). Lignin: occurrence, biogenesis and biodegradation. Annu. Rev. Plant Physiol. Plant Mol. Biol. 41, 455-496. doi: 10.1146/annurev.pp.41.060190.002323

Livak, K. J., and Schmittgen, T. D. (2001). Analysis of relative gene expression data using real-time quantitative PCR and the $2^{-\Delta \Delta C T}$ method. Methods 25 , 402-408. doi: 10.1006/meth.2001.1262

McCarthy, R. L., Zhong, R., and Ye, Z. H. (2009). MYB83 is a direct target of SND1 and acts redundantly with MYB46 in the regulation of secondary cell wall biosynthesis in Arabidopsis. Plant Cell Physiol. 50, 1950-1964. doi: $10.1093 / \mathrm{pcp} / \mathrm{pcp} 139$

McCartney, L., Marcus, S. E., and Knox, J. P. (2005). Monoclonal antibodies to plant cell wall xylans and arabinoxylans. J. Histochem. Cytochem. 53, 543-546. doi: 10.1369/jhc.4B6578.2005

Mitsuda, N., Seki, M., Shinozaki, K., and Ohme-Takagi, M. (2005). The NAC transcription factors NST1 and NST2 of Arabidopsis regulate secondary wall thickenings and are required for anther dehiscence. Plant Cell 17, 2993-3006. doi: $10.1105 /$ tpc. 105.036004

Orfila, C., Sorensen, S. O., Harholt, J., Geshi, N., Crombie, H., Truong, H. N., et al. (2005). QUASIMODO1 is expressed in vascular tissue of Arabidopsis thaliana inflorescence stems, and affects homogalacturonan and xylan biosynthesis. Planta 222, 613-622. doi: 10.1007/s00425-005-0008-Z

Pattathil, S., Avci, U., Baldwin, D., Swennes, A. G., McGill, J. A., Popper, Z., et al. (2010). A comprehensive toolkit of plant cell wall glycan-directed monoclonal antibodies. Plant Physiol. 153, 514-525. doi: 10.1104/pp.109.151985

Pattathil, S., Avci, U., Miller, J. S., and Hahn, M. G. (2012). Immunological approaches to plant cell wall and biomass characterization: glycome profiling. Methods Mol. Biol. 908, 61-72. doi: 10.1007/978-1-61779-956-3 6

Pattathil, S., Harper, A. D., and Bar-Peled, M. (2005). Biosynthesis of UDPxylose: characterization of membrane-bound AtUxs2. Planta 221, 538-548. doi: 10.1007/s00425-004-1471-7

Pedrioli, P. G., Eng, J. K., Hubley, R., Vogelzang, M., Deutsch, E. W., Raught, B., et al. (2004). A common open representation of mass spectrometry data and its application to proteomics research. Nat. Biotechnol. 22, 1459-1466. doi: $10.1038 /$ nbt1031

Peña, M. J., Zhong, R., Zhou, G. K., Richardson, E. A., O’neill, M. A., Darvill, A. G., et al. (2007). Arabidopsis irregular xylem8 and irregular xylem9: implications for the complexity of glucuronoxylan biosynthesis. Plant Cell 19, 549-563. doi: 10.1105/tpc.106.049320

Persson, S., Caffall, K. H., Freshour, G., Hilley, M. T., Bauer, S., Poindexter, P., et al. (2007). The Arabidopsis irregular xylem 8 mutant is deficient in glucuronoxylan and homogalacturonan, which are essential for secondary cell wall integrity. Plant Cell 19, 237-255. doi: 10.1105/tpc.106.047720
Persson, S., Wei, H., Milne, J., Page, G. P., and Somerville, C. R. (2005). Identification of genes required for cellulose synthesis by regression analysis of public microarray data sets. Proc. Natl. Acad. Sci. U.S.A. 102, 8633-8638. doi: 10.1073/pnas.0503392102

Petersen, P. D., Lau, J., Ebert, B., Yang, F., Verhertbruggen, Y., Kim, J. S., et al. (2012). Engineering of plants with improved properties as biofuels feedstocks by vessel-specific complementation of xylan biosynthesis mutants. Biotechnol. Biofuels 5:84. doi: 10.1186/1754-6834-5-84

Raes, J., Rohde, A., Christensen, J. H., Van De Peer, Y., and Boerjan, W. (2003). Genome-wide characterization of the lignification toolbox in Arabidopsis. Plant Physiol. 133, 1051-1071. doi: 10.1104/pp.103.026484

Sangha, A. K., Parks, J. M., Standaert, R. F., Ziebell, A., Davis, M., and Smith, J. C. (2012). Radical coupling reactions in lignin synthesis: a density functional theory study. J. Phys. Chem. B 116, 4760-4768. doi: 10.1021/Jp2122449

Scheller, H. V., and Ulvskov, P. (2010). Hemicelluloses. Annu. Rev. Plant Biol. 61, 263-289. doi: 10.1146/annurev-arplant-042809-112315

Schilmiller, A. L., Stout, J., Weng, J. K., Humphreys, J., Ruegger, M. O., and Chapple, C. (2009). Mutations in the cinnamate 4-hydroxylase gene impact metabolism, growth and development in Arabidopsis. Plant J. 60, 771-782. doi: 10.1111/j.1365-313X.2009.03996.x

Shao, M., Zheng, H., Hu, Y., Liu, D., Jang, J. C., Ma, H., et al. (2004). The GAOLAOZHUANGREN1 gene encodes a putative glycosyltransferase that is critical for normal development and carbohydrate metabolism. Plant Cell Physiol. 45, 1453-1460. doi: 10.1093/pcp/pch168

Steiner-Lange, S., Unte, U. S., Eckstein, L., Yang, C., Wilson, Z. A., Schmelzer, E., et al. (2003). Disruption of Arabidopsis thaliana MYB26 results in male sterility due to non-dehiscent anthers. Plant J. 34, 519-528. doi: 10.1046/j.1365313X.2003.01745.x

Sterling, J. D., Atmodjo, M. A., Inwood, S. E., Kumar Kolli, V. S., Quigley, H. F., Hahn, M. G., et al. (2006). Functional identification of an Arabidopsis pectin biosynthetic homogalacturonan galacturonosyltransferase. Proc. Natl. Acad. Sci. U.S.A. 103, 5236-5241. doi: 10.1073/pnas.0600120103

Sterling, J. D., Lemons, J. A., Forkner, I. F., and Mohnen, D. (2005). Development of a filter assay for measuring homogalacturonan: $\alpha$ (1,4)-galacturonosyltransferase activity. Anal. Biochem. 343, 231-236. doi: 10.1016/j.ab.2005.05.037

Sykes, R., Kodrzycki, B., Tuskan, G., Foutz, K., and Davis, M. (2008). Within tree variability of lignin composition in Populus. Wood Sci. Technol. 42, 649-661. doi: 10.1007/s00226-008-0199-0

Tan, L., Eberhard, S., Pattathil, S., Warder, C., Glushka, J., Yuan, C., et al. (2013). An Arabidopsis cell wall proteoglycan consists of pectin and arabinoxylan covalently linked to an arabinogalactan protein. Plant Cell 25, 270-287. doi: $10.1105 /$ tpc. 112.107334

Taylor, N. G., Howells, R. M., Huttly, A. K., Vickers, K., and Turner, S. R. (2003). Interactions among three distinct CesA proteins essential for cellulose synthesis. Proc. Natl. Acad. Sci. U.S.A. 100, 1450-1455. doi: 10.1073/pnas.03376 28100

Thevenin, J., Pollet, B., Letarnec, B., Saulnier, L., Gissot, L., Maia-Grondard, A., et al. (2011). The simultaneous repression of CCR and CAD, two enzymes of the lignin biosynthetic pathway, results in sterility and dwarfism in Arabidopsis thaliana. Mol. Plant 4, 70-82. doi: 10.1093/mp/ssq045

Wang, Y., Zhang, W. Z., Song, L. F., Zou, J. J., Su, Z., and Wu, W. H. (2008). Transcriptome analyses show changes in gene expression to accompany pollen germination and tube growth in Arabidopsis. Plant Physiol. 148, 1201-1211. doi: 10.1104/pp.108.126375

Weatherly, D. B., Atwood, J. A. 3rd., Minning, T. A., Cavola, C., Tarleton, R. L., and Orlando, R. (2005). A Heuristic method for assigning a false-discovery rate for protein identifications from Mascot database search results. Mol. Cell. Proteomics 4, 762-772. doi: 10.1074/mcp.M400215-MCP200

Weng, J. K., Mo, H., and Chapple, C. (2010). Over-expression of F5H in COMT-deficient Arabidopsis leads to enrichment of an unusual lignin and disruption of pollen wall formation. Plant J. 64, 898-911. doi: 10.1111/j.1365313X.2010.04391.x

Wilson, Z. A., Song, J., Taylor, B., and Yang, C. (2011). The final split: the regulation of anther dehiscence. J. Exp. Bot. 62, 1633-1649. doi: 10.1093/jxb/err014

Yamaguchi, M., Goue, N., Igarashi, H., Ohtani, M., Nakano, Y., Mortimer, J. C., et al. (2010). VASCULAR-RELATED NAC-DOMAIN6 and VASCULARRELATED NAC-DOMAIN7 effectively induce transdifferentiation into xylem 
vessel elements under control of an induction system. Plant Physiol. 153, 906-914. doi: 10.1104/pp.110.154013

Yang, C., Xu, Z., Song, J., Conner, K., Vizcay Barrena, G., and Wilson, Z. A. (2007). Arabidopsis MYB26/MALE STERILE35 regulates secondary thickening in the endothecium and is essential for anther dehiscence. Plant Cell 19, 534-548. doi: 10.1105/tpc.106.046391

Yin, Y., Chen, H., Hahn, M. G., Mohnen, D., and Xu, Y. (2010). Evolution and function of the plant cell wall synthesis-related glycosyltransferase family 8. Plant Physiol. 153, 1729-1746. doi: 10.1104/pp.110. 154229

York, W. S., and O'Neill, M. A. (2008). Biochemical control of xylan biosynthesis-which end is up? Curr. Opin. Plant Biol. 11, 258-265. doi: 10.1016/j.pbi.2008.02.007

Yuan, T. Q., Sun, S. N., Xu, F., and Sun, R. C. (2011). Characterization of lignin structures and lignin-carbohydrate complex (LCC) linkages by quantitative ${ }^{13} \mathrm{C}$ and 2D HSQC NMR spectroscopy. J. Agric. Food Chem. 59, 10604-10614. doi: $10.1021 /$ jf2031549

Zhong, R., Peña, M. J., Zhou, G. K., Nairn, C. J., Wood-Jones, A., Richardson, E. A., et al. (2005). Arabidopsis Fragile Fiber8, which encodes a putative glucuronyltransferase, is essential for normal secondary wall synthesis. Plant Cell 17, 3390-3408. doi: 10.1105/tpc.105.035501

Zou, J., Song, L., Zhang, W., Wang, Y., Ruan, S., and Wu, W. H. (2009). Comparative proteomic analysis of Arabidopsis mature pollen and germinated pollen. J. Integr. Plant Biol. 51, 438-455. doi: 10.1111/j.1744-7909.2009. 00823.x

Conflict of Interest Statement: The authors declare that the research was conducted in the absence of any commercial or financial relationships that could be construed as a potential conflict of interest.

Received: 29 May 2014; paper pending published: 20 June 2014; accepted: 03 July 2014; published online: 28 July 2014.

Citation: Hao Z, Avci U, Tan L, Zhu X, Glushka J, Pattathil S, Eberhard S, Sholes T, Rothstein GE, Lukowitz W, Orlando R, Hahn MG and Mohnen D (2014) Loss of Arabidopsis GAUT12/IRX8 causes anther indehiscence and leads to reduced $G$ lignin associated with altered matrix polysaccharide deposition. Front. Plant Sci. 5:357. doi: 10.3389/fpls.2014.00357

This article was submitted to Plant Physiology, a section of the journal Frontiers in Plant Science.

Copyright (c) 2014 Hao, Avci, Tan, Zhu, Glushka, Pattathil, Eberhard, Sholes, Rothstein, Lukowitz, Orlando, Hahn and Mohnen. This is an open-access article distributed under the terms of the Creative Commons Attribution License (CC BY). The use, distribution or reproduction in other forums is permitted, provided the original author(s) or licensor are credited and that the original publication in this journal is cited, in accordance with accepted academic practice. No use, distribution or reproduction is permitted which does not comply with these terms. 